\title{
Rheology-Morphology Interrelationships for Nanocomposites based on Polymer Matrices
}

\author{
Valery Kulichikhin ${ }^{1}$, Alexander Semakov르, Valery Karbushev ${ }^{1}$, \\ Veronica Makarova1, Eduardo Mendes², \\ Hartmut Fisher ${ }^{2}$ and Stephen Picken ${ }^{2}$ \\ ${ }^{1}$ A.V.Topchiev Institute of Petrochemical Synthesis, Russian Academy of Sciences, \\ ${ }^{2}$ Delft University of Technology, \\ ${ }^{1}$ Russia \\ 2The Netherlands
}

\section{Introduction}

Nanocomposites based on polymer matrices are heterogeneous systems containing nanodispersed fillers distributed in polymer. The main aim of developing this kind of materials is to reinforce commodity thermoplastics giving them some functional properties (low flammability, increased barrier behavior, higher heat deflection temperature, and so on). When fillers are nanoscopic, there are advantages afforded to filled polymers and composites that lead to performance enhancements. These advantages results primarily from filler size reduction and the concomitant increase in interface. This means, that development of materials with unique or improved properties does not demand creation of new chemical manufactures for production of new and, as a rule, expensive polymers. For this reason the high activity of various research groups in the field of detail investigation of structure and properties of nanoparticles of different nature and composite materials on their base is being observed.

The main portion of this activity is devoted to search and detail characterization of nanoparticles of various nature (nanoclays, nanodiamonds, metals and their oxides, salts and specific compounds, for example CdSe, playing the role of quantum dots, and others). Remaining fillers for specific functional application outside the scope of this Chapter, we intend to consider here very simple nanofillers, such as clays and nanodiamonds serving for the development of a new family of engineering plastics. At literature analysis it is possible to understand that a lot of has been devoted to the attention elaboration of preparation methods, their characterization by different methods, and mechanical properties of final products (see, for example, Pinnovaia \& Beall, 2000). Meanwhile, approaches to compatibility of particles and polymer, as well as their processing, remain on "periphery of main stream". We would like to fill this gap by information based on our experience, i.e., to consider some methods of compatibility of particles with polymers, rheological properties of nanocomposites precursors, interrelationships between rheology and morphology for heterophase viscoelastic media and approaches to their processing.

The crucial question appearing at realization of nanocomposite concept consists in: "Why nanofillers reinforce polymer with a high efficacy?" There are different versions of answer 
this question. All of them are based on superhigh interfaces in such systems, but the angle of vision is different. In our opinion, the main reinforcing effect comes from polymers due to the absorption of macromolecules onto external (e.g. nanodiamonds) or internal (intercalated layered silicates) surfaces of nanoparticles, realization of their "non-natural" conformations, formation of dense adsorption layers, separation of conformations between volume and adsorbed layers. This proceeds at melt mixing of particles with polymer. Solidification of precursors by cooling (thermoplastics) or crosslinking (reactive resins) leads to "freezing" these conformations for amorphous polymers or to specific crystallization behavior for semi-crystalline polymers. The term "specificity" in this case means that depending on macromolecular conformations in liquid precursor the final crystalline structure of polymer in solid nanocomposite will be changed.

It other words, the introduction of nanodimensional particles modifies the structure of polymer. All these events take place at minor content of nanofillers, less than percolation threshold. At higher concentration only a possibility to form by particles the arming network appears but this is another story.

Based on above mentioned concept, the reinforcing effect should be especially expressed at homogeneous distribution of particles, but all of them due to high interfacial energy and presence on the surface of functional groups (e.g., nanodiamonds of detonation synthesis) inclined to form aggregates. That is why, the task number one is to introduce minor content of particles to polymer as possible homogeneously for distribute them to avoid large aggregates. It would be fine, if disaggregation procedure could be involved to compatibility process. We will consider various methods of compatibility tested by our group on an example of nanodiamonds keeping in mind above written requirements. The task number two consists in detail investigation of rheology-morphology interrelationships that is extremely important for all heterophase systems, because flow may influence significantly on nanoparticles distribution. Since flow is obligatory factor of processing, the distribution of particles reached at mixing could be changed during processing. The task number three relates to rheology-structure interrelationships where the term "structure" concerns crystallographic level as polymer, as nanofiller. The possible correlations between rough morphological and fine structure levels should be estimated. This is very important for final properties of nanocomposites, and among them mechanical characteristics are dominant. All these tasks will be considered in this Chapter.

\section{Objects}

As a matrix, series of polymers of different polarities have been used: hydrophilic hydroxypropylcellulose (HPC), capable to form liquid crystal (LC) phase as in melt, as in solution, styrene-acrylonitrile copolymer (SAN), polysulfone (PSF), polyisobutylene (PIB), and others.

The majority of experiments were carried out on two kinds of nanofillers: Namontmorillonite (Cloisite $\mathrm{Na}+$ ) and organoclays (e.g., Cloisite 15A) produced by Southern Clay Products, USA, and nanodiamonds (ND) of detonation synthesis (Shenderova, 2004; Detkov et al.). ND were prepared by "Electrochimpribor", Russia (trade mark "PUOO-SH $96 ")$, weight fraction of ND in a powder is not less than $98 \%$, density is $3.3 \mathrm{~g} / \mathrm{cm} 3$.

In spite of the fact that individual particles of both fillers have nanodimensions, there exist aggregates of micron size in the powder. The main problem here is to find the appropriate method of combining particles with polymer. 
Four methods have been tested:

1. Traditional mechanical mixing at temperature higher melting or softening points ("standard mixing") (Karbushev et al., 2007);

2. Melt mixing at very high shear rates, i.e., in conditions close to elastic turbulence and instability of melt flow as whole (Vinogradov \& Malkin, 1966; Malkin, 2006; Karbushev et al., 2008), where turbulent vortexes and probable cavitation zones could induce disaggregation of large particles;

3. Deposition of particles on a surface of polymer grains distributed in inert liquid medium at ultrasonic action with subsequent removing inert liquid by filtration and drying nanocomposite precursor (Konstantinov et al., 2009; Karbushev et al., 2009);

4. Introducing of particles to polymer solution at sonication with subsequent removing a solvent (so-called "solution method") (Karbushev et al., 2007).

The first method, the most suitable for thermoplastics, was used as the reference method. It was realized on laboratory mixer of screw-plunger type with operating volume of 4-5 ml (Fig.1). Mixing procedure takes $7 \mathrm{~min}$ at temperatures $135-150^{\circ} \mathrm{C}(\mathrm{HPC}), 200-220^{\circ} \mathrm{C}$ (SAN) and $280-300^{\circ} \mathrm{C}$ (PSF). Rotation of screw is around $60-80 \mathrm{rpm}$ and nanocomposite precursor circulates not only in radial, but also in axial directions. Then, plug 6 was avoided, and the filled melt extrudes through a capillary with $1 / d=20 / 1(\mathrm{~mm})$.

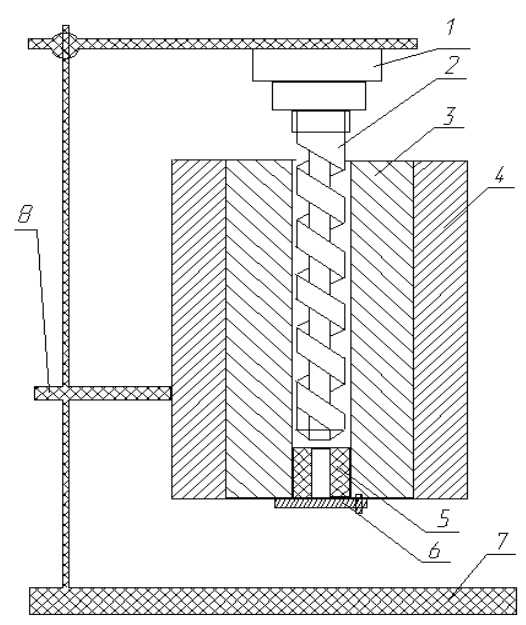

Fig. 1. Scheme of screw-plunger mixer: 1- engine; 2 - screw; 3 - working chamber; 4 - electric furnace; 5 - capillary; 6 - catch gear (plug); 7 - baseplate; 8 - holder.

The second mixing method is also based on a direct combining of particles with polymer melt, but at super-high shear rates, i.e., in a regime of elastic instability. The same apparatus as before has been used but fast rotation of a screw $(600-800 \mathrm{rpm})$ allowed us to reach shear stresses of $10^{5}-10^{6} \mathrm{~Pa}$ for SAN and $10^{6}-10^{7} \mathrm{~Pa}$ for PSF. According to classical papers of Vinogradov et al. (Vinogradov et al., 1966; Vinogradov \& Ivanova, 1968), at such conditions so-called "spurt" takes place, i.e., abnormal increase of shear rate at approximately constant shear stress. "Spurt" was explained as relaxation transition of melt to rubber-like state induced by shear, loosing of tack to the wall of capillary and slippage. Polymer in a slippage step relaxes to melt state and becomes tacky again, sticking to the wall. Repeating cycles of 
"stick-slip" leads to appearance of defects of various topology on the extrudate surface: shark-skin, spirals, waves, and so on.

Subsequent numerous investigations have considered this phenomenon in details under different vision angles, and suggested some new mechanisms and driving forces (Yarin \& Graham, 1998; Yang et al. 1998), but for the aim of this Chapter the important feature is irregularity of a stream only. It is a "tool" we tried to use to disaggregate large particles formed by nanoparticles connected by dispersion interactions Van-der-Waals and electrostatic forces.

The "colloidal-deposition" method consisted in disaggregation and dispersion of the filler particles in a low-molecular-weight liquid medium, inert towards the polymeric matrix, subjected to the action of an ultrasonic field. A polymer powder was added to the polymer suspension without terminating the intermittent sonication. Under these conditions, the socalled heteroadagulation occurred, e.g., nanodiamond particles are deposited (immobilized) onto the surface of coarser polymer particles. As the process was complete, the solid phase was separated by filtration, dried, and nanocomposite precursors were processed by extrusion or hot pressing. Because of the lack of analogies, this way to produce nanocomposites was named as "colloidal-deposition" method.

Several composites were obtained by popular laboratory method via polymer solutions. For this purpose, the filler was dispersed in a polymer solution under the action of ultrasound. We used $4 \%$ solutions of HPC in distilled water, SAN in acetone, and PSF in chloroform. In this case, composite films were produced by casting, followed by evaporation of the solvent. Thin slices of nanocomposite precursors prepared according above mentioned methods were cut and characterized by optical and electron-microscopical methods. The special program of digital images analysis has been developed in frames of MathLab 7.0. It was very significant step to analyze dimensions of particles directly in polymer matrices, but not in model dispersions. Optical images of nanocomposites containing $1 \%$ of ND in SAN matrix are shown in Fig.2.

As is seen, the homogeneity of particles distribution and their dimensions depend essentially on mixing method. The best results were achieved by mixing in "spurt" regime and via stage of colloidal deposition. The distribution curves in optical domain of dimensions are presented in Fig.3.

Maximum of distribution curves for all blends is located in the dimension region of 1-2 $\mathrm{mkm}$, but its sharpness is different. The most narrow maxima are observed for "spurt" regime and method of colloidal deposition. For standard mechanical mixing and solution method more flat maxima and presence of large agglomerates are observed.

For estimation of dispersity degree in sub-micron domain the transmission electron microscopy has been used. Micrographs of specimens with different magnification one can see in Figs 4 and 5. From the qualitative view point samples obtained via "spurt" and "colloidal deposition" methods are much better than the reference sample.

The quantitative analysis has shown that melt mixing in "spurt" regime is the best: the maximum fraction of particles is located around $\sim 40 \mathrm{~nm}$ while for colloidal deposition method its position is $80-120 \mathrm{~nm}$. The generalized polydisperisty curve for nanocomposite SAN-1\% of ND is presented in Fig. 6

All set of data shows that melt mixing in "spurt" regime is the best and looks like very attractive for application, because high-speed mixers can be used and the process does not contain any solvent that should be recuperated and regenerated. An attempt to reach 

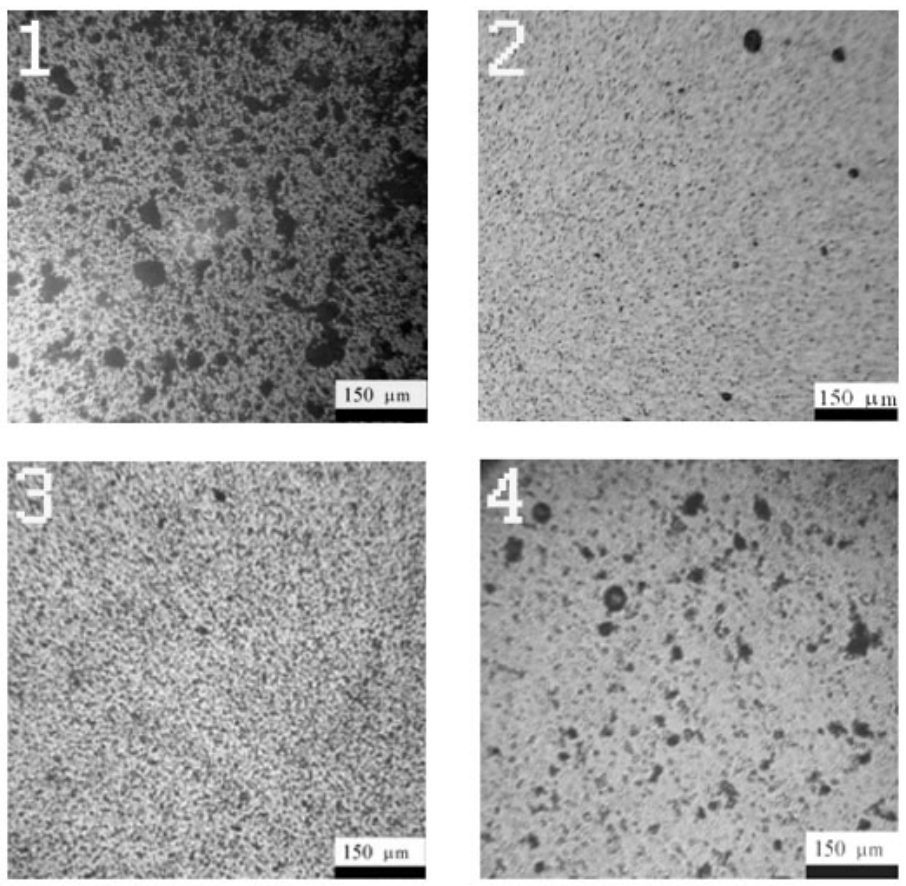

Fig. 2. Micrographs of SAN - $1 \mathrm{wt} \% \mathrm{ND}$ blends prepared by: "standard melt mixing" (1); melt-mixing in "spurt" regime (2); "colloidal-deposition" method (3); d) solvent method (4).

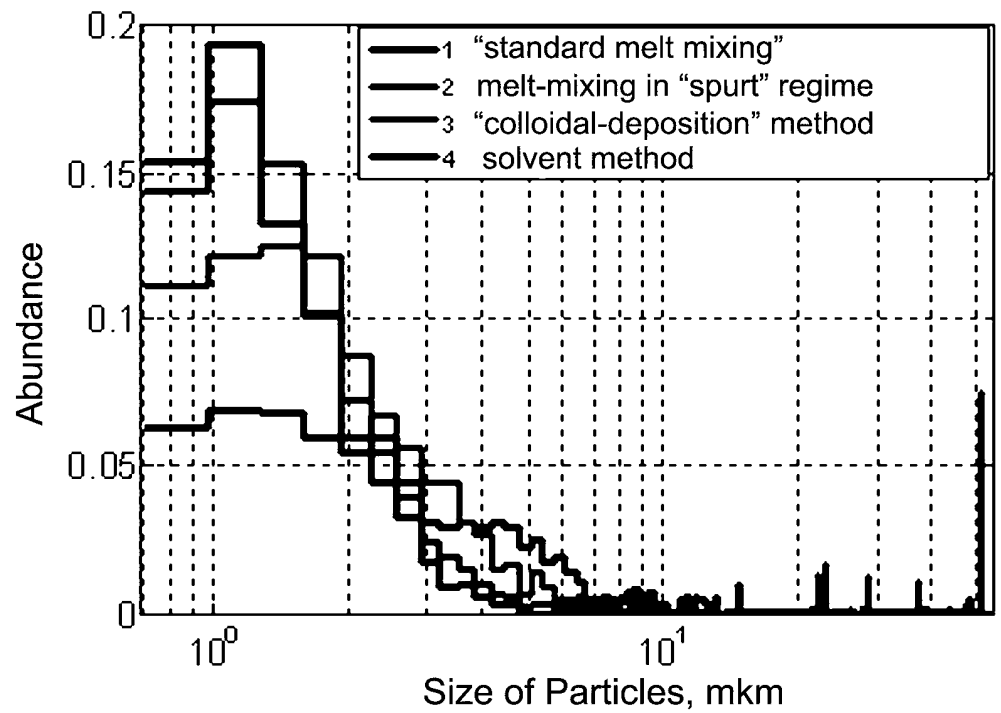

Fig. 3. Dimensional distribution of nanodiamond particles in SAN (1\%) for different mixing methods (optical diapason). 

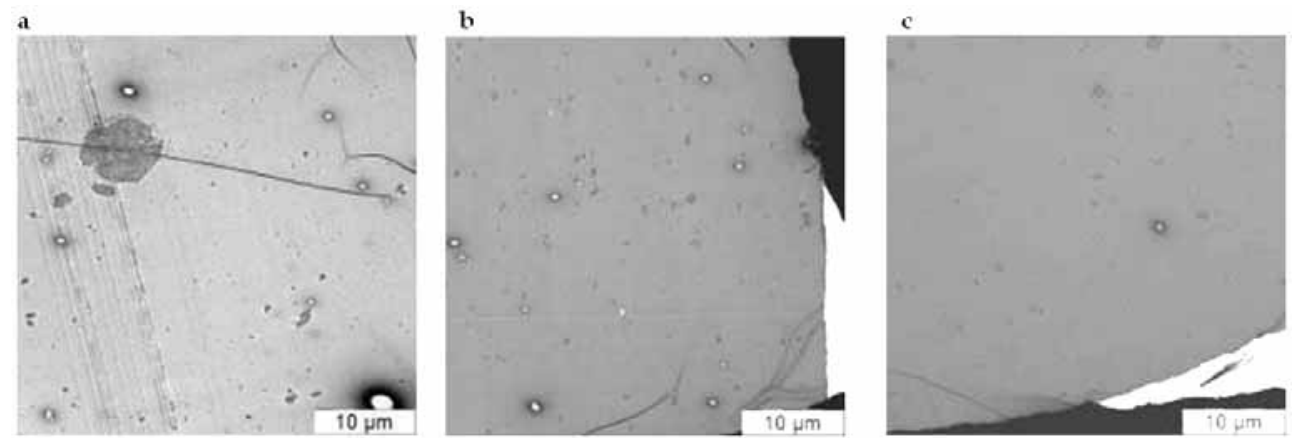

Fig. 4. Micrographs of composites SAN/1\% of ND prepared by "standard mechanical mixing" (a), colloidal-deposition method (b) and in "spurt" regime (c).

a b

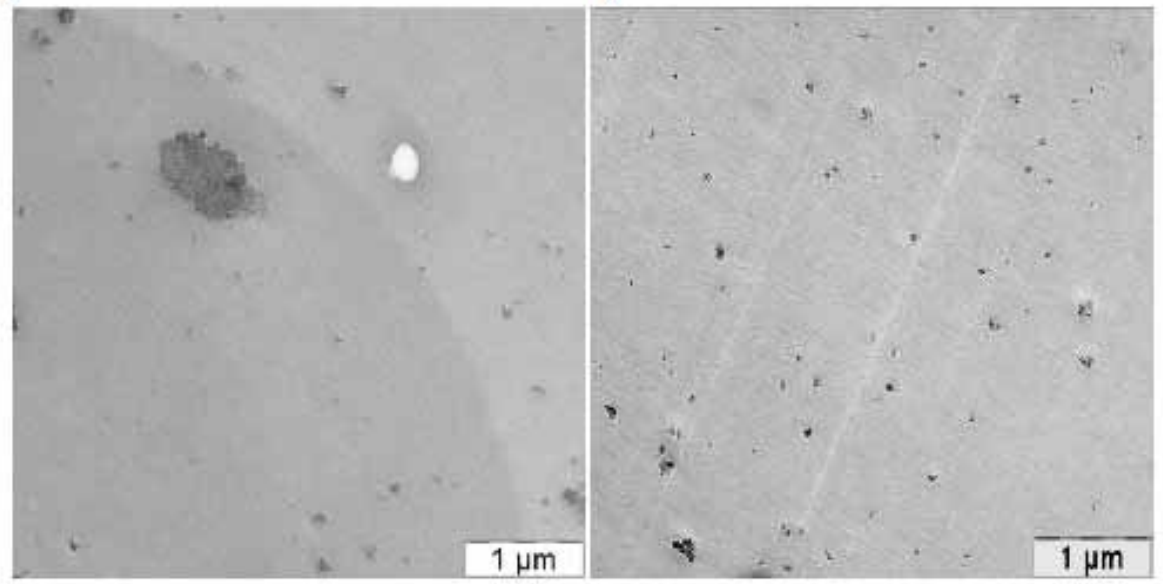

Fig. 5. TEM images of composites SAN/1 wt.\% ND obtained by standard melt-mixing (a) and mixing in "spurt" regime (b).

smaller particles of ND in polymer matrix was done via chemical blocking the surface functional groups presented in ND. Two reagents were tested for this procedure: trifluoromethansulfoacid (TFSA) and hexafluoroisopropanol (HFIP). In this case we cannot avoid organic solvent because dispersion of $1 \mathrm{~g} \mathrm{ND}$ in $100 \mathrm{ml}$ of hexane is treated by ultrasound, then $1 \mathrm{ml}$ of TFSA or HFIP is added under permanent sonication. After deposition of particles, hexane was removed by decantation and $100 \mathrm{ml}$ of acetone was added to deposit for washing. After subsequent agitation and centrifuging the powder was washed with distilled water and dried in exsiccator.

The most suitable method for combining modified ND particles with polymer is colloidal deposition since TFSA and HFIP could be added to inert solvent used as a medium for deposition of ND onto polymer grains. Visually, homogeneous by dimensions ND particles are absorbed by polymer surface. This method of compatibility will be considered again at analysis of mechanical properties of nanocomposites. 


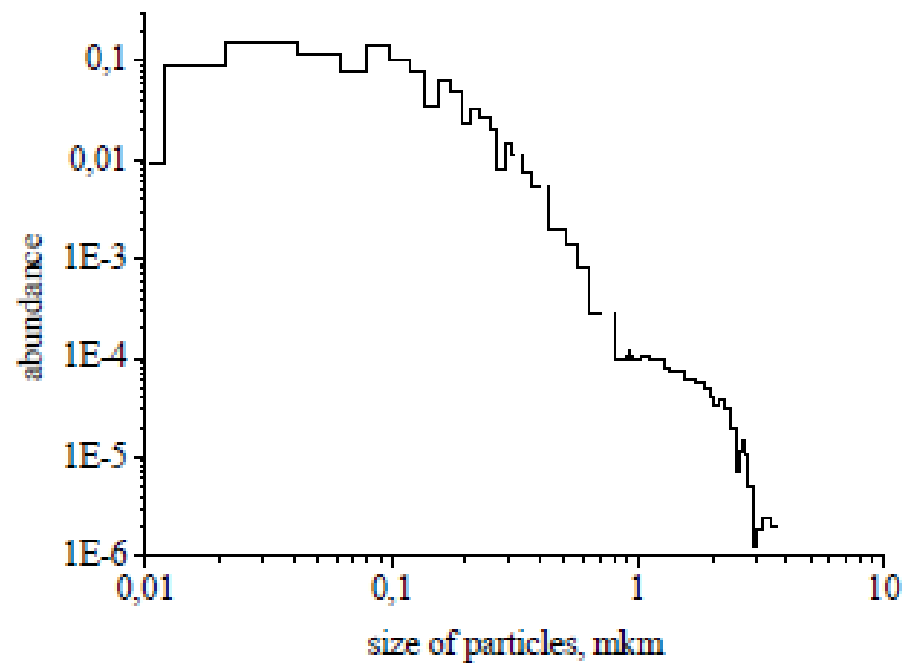

Fig. 6. Generalized polydispersity curve for SAN/1 wt\% ND composite prepared in "spurt" regime.

\section{Methods}

\subsection{Rheological properties}

Both capillary and rotation equipment was used for measuring rheological properties: rotation rheometer "Rheostress 600" ("Thermo Haake", Germany) with operating units cone-plate and plate-plate, as well as capillary rheometer MV-3M of melt-indexer type (V.E.Dreval et al. 1995). As a rule, appearance of samples was analyzed visually after dismounting operating units after every experiment ("post-factum" mode).

Visualization of stream is very important for such heterophase systems as nanocomposites. The matter is that very often we think about stability of the distribution character at processing compared with precursors. But our experience shows that it is not true and shear field can change morphology of nanocomposite significantly. For this analysis the transparent cell of "lens-plate" allowing us to visualize shear stream in transmitting nonpolarized and polarized light was designed and constructed. The scheme of this operating unit is presented in Fig.7a. Applied web-camera allows us to see evolution of morphology at flow. Knowing angular speed of plate rotation $\omega$, curvature radius of lens $(R=50 \mathrm{~mm})$, the gap between lens apex and plate $h$ the shear rate was calculated

$$
\dot{\gamma}(r)=\frac{2 R r}{r^{2}+h} \omega
$$

as a function of $r$. This dependence has maximum

$$
\dot{\gamma}_{\max }=\omega \frac{\sqrt{2 R h}}{2 h}
$$

at $r=\sqrt{2 R h}$. If the gap is absent the dependence becomes by hyperbolic function: 


$$
\dot{\gamma}(r)=\frac{2 R}{r} \omega
$$

Actually, this apparatus scans a medium under investigation at constant angular velocity of plate.

In addition, based on this equipment the two-beam interferometer serving for visualization of stream morphology features for elastic polymer melts, solutions and nanocomposites, non-visible in transmitting light (details of modulation of refraction index of medium as a result of photoelasticity) was designed (Fig.7b).

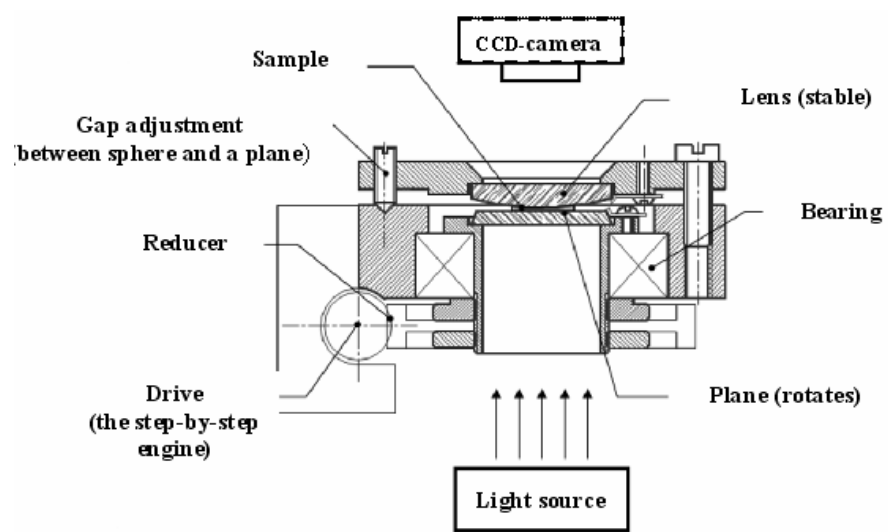

(a)

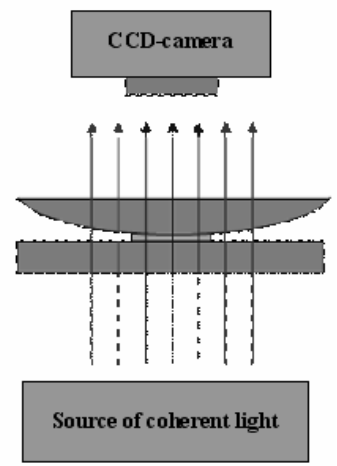

(b)

Fig. 7. Scheme of equipment for visualization of stream in axially-symmetric shear field (a); scheme of interferometer (b). See explanations in text.

\subsection{Rheo-X-ray}

For rheo-X-ray measurements the following equipment has been used (S. Viale et al. 2005; Ruijter et al., 2006). Two glass coaxial capillaries with radii $R_{\text {out }}=0.5$ (outer, unmoved) and $\mathrm{R}_{\mathrm{in}}=0.25$ (inner, movable) and a length of $\sim 10 \mathrm{~cm}$ combine the Couette cell (Fig.8a). The shear rate in the narrow gap, $\dot{\gamma}$ was calculated as

$$
\dot{\gamma}=r \frac{d \omega}{d r}=\frac{\omega \bar{R}}{R_{\text {out }}-R_{\text {in }}}=\frac{2 \pi n\left(R_{\text {out }}-R_{\text {in }}\right) / 2}{R_{\text {out }}-R_{\text {in }}},
$$

where $\omega$ is angular velocity and $n$ is rotation speed (rpm). For the present geometry $\dot{\gamma}=0,157 n$.

The scheme of the experimental set up is shown in Fig. 8b. The Couette cell was adjusted relative to the collimator and $2 \mathrm{D}$ detector by means of the $\mathrm{x}, \mathrm{y}, \mathrm{z}$-stage of the goniometer where using the alignment laser beam and video camera. The collimated X-ray beam can pass either through the centre axis of the Couette geometry (passing two gaps along the gradient axis) or tangential to the gap along the streamlines. In our experiments we mainly used the first option with the beam along the gradient direction. In all cases the differential $X$-ray diffractograms (background was capillaries filled with water) were detected. The diameter of the incident $X$-ray beam used was $0.5 \mathrm{~mm}$. 


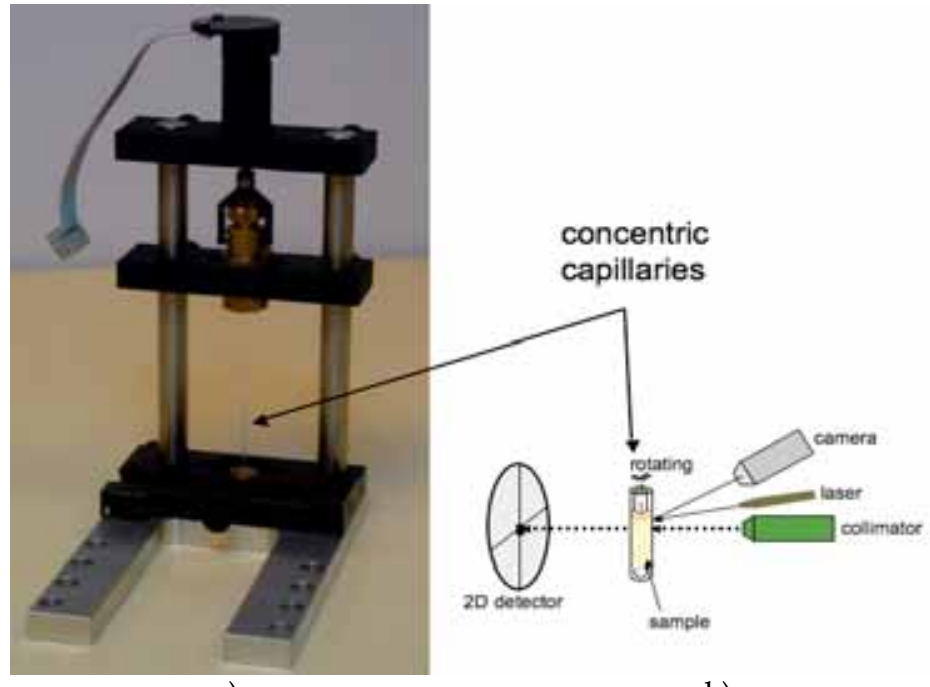

a)

b)

Fig. 8. Image of the micro-Couette used in the experiment (a); scheme of experimental device for "rheo-X-ray" measurements (b).

The overall orientation can be expressed quantitatively in terms of the orientation parameter $<\bar{P}_{2}>$ that is calculated as follows. The intensity distribution can be described as

$$
I=I_{0}+A e^{\alpha \cos ^{2}\left(\phi-\phi_{0}\right)}
$$

where $\varphi$ is the azimuthal angle, $\alpha$ is a parameter characterizing the width of intensity distribution. Knowing $\alpha$, we can calculate $<\bar{P}_{2}>$ as

$$
\left\langle\bar{P}_{2}\right\rangle=\frac{\int_{-1}^{1} P_{2}(\cos \phi) e^{\alpha \cos ^{2} \phi} d \cos \phi}{\int_{-1}^{1} e^{\alpha \cos ^{2} \phi} d \cos \phi}
$$

\subsection{Mechanical and thermomechanical properties}

Mechanical properties of extruded samples of the circle cross-section were measured on tensile machine Instron 1122 at extension rate of $10 \mathrm{~mm} / \mathrm{min}$.

Notched samples of PSF/ND composites have been tested for Izod impact strength by standard method.

\subsection{Tribological properties}

By means of an original technique the sliding friction coefficient and wear resistance of composites relatively nichrome wire has been measured. Principle of tribometer action is the following: in the holder of horizontally located microdrill the tested sample (extrudate) 
contacting with the counterface (nichrome wire, $\mathrm{d}=50 \mathrm{mkm}$ ) on whole radius (angle of contact is $2 \Pi$ ) was fixed. Rpm of the microdrill was chosen corresponding to the linear speed of wire sliding of $1 \mathrm{~m} / \mathrm{min}$. The friction coefficient was calculated using Euler's formula

$$
S_{0}=S e^{f \alpha}
$$

where $\mathrm{S}_{0}$ - loading, $\mathrm{S}$ - strain, $f$ - friction coefficient, a - angle of contact of specimen with wire.

\section{Results and discussion}

\subsection{Rheology and stream morphology}

Development of morphology at flow (in-situ mode) was studied on model system: viscoelastic solution of PIB in cetane filled with rather large $(\mathrm{d} \sim 80 \mathrm{mkm})$ monodisperse spherical particles of PMMA. For matrix solution normal stresses start to exceed tangential stresses (Weissenberg number, $\mathrm{Wi}>1$ ) at shear rate of $0.2 \mathrm{~s}^{-1}$ as is seen from dependences of stresses and viscosity on shear rate (Fig.9).

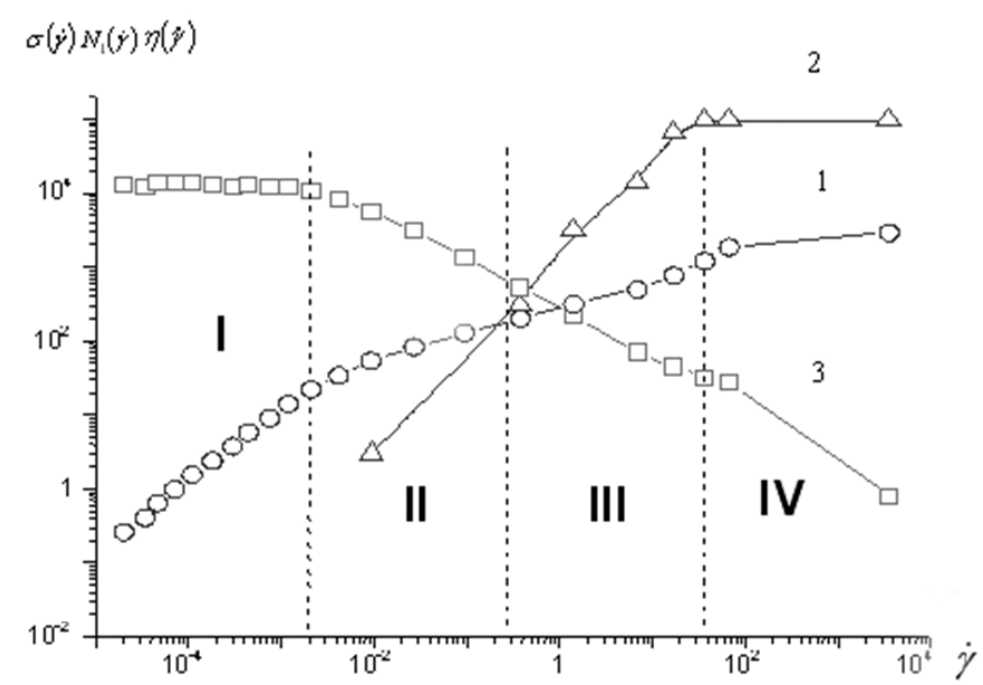

Fig. 9. Dependences $\sigma(\dot{\gamma})-1, N_{1}(\dot{\gamma})-2, \eta(\dot{\gamma})-3$ for PIB solution in cetane.

This is important point where elastic response of solution becomes dominant. $5 \%$ of PMMA microspheres were introduced to this solution, a drop of composition was placed in the center of plate and lens of the apparatus as shown in Fig.7a, and squeezed it up to formation of desired gap width (in this case $\mathrm{h}=200 \mathrm{mkm}$ ). Shooting via microscope with partially crossed polars was done just to see simultaneously morphology and optical anisotropy of a matrix at flow. A series of frames from a movie illustrating the evolution of morphology in axially symmetric shear field in time at angular speed of $1 \mathrm{rad} / \mathrm{s}$ is shown in Fig.10.

The initially chaotic distribution of PMMA spheres in PIB solution is gradually replaced by ordering of particles on a background of Maltese cross indicating on optical anisotropy of photoelastic matrix initiated by shear. Particles participate in a curve motion forming first 

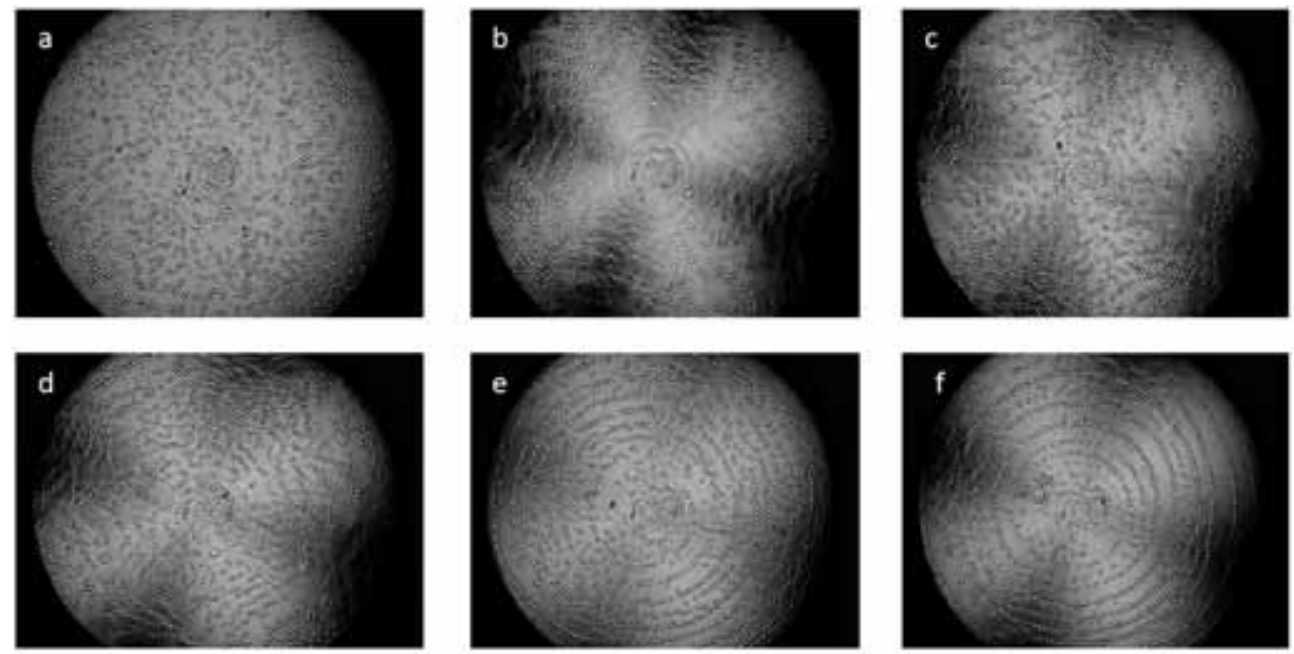

Fig. 10. Evolution of stream morphology for PIB solution filled with 5\% of PMMA spheres. Time increases from a) to f).

short chains and then extended arches, and at last the closed circles. Ordering process looks like formation of chains with closed package of particles along streamlines. It is important to note that there appear alternating layers along radius enriched by particles. In other words, stratification of stream takes place and initially chaotic distribution of particles is replaced by their ordering. Since there is a gap between lens and plate, the radial profile of shear rate has a maximum. In the center the rate as well as the stress is equal to zero, then it increases up to a distance of $4.5 \mathrm{~mm}\left(\dot{\gamma}=10 \mathrm{~s}^{-1}\right)$, then decreases monotonically up to zero again. The vision area on frames is approximately $3 \mathrm{~mm}$, and this means that on periphery of frames the shear rate is maximum $\left(\dot{\gamma}=9.8 \mathrm{~s}^{-1}\right)$. In the central zone there is no ordering of particles, and chains of particles appear at a distance of $0.2 \mathrm{~mm}$, at $\dot{\gamma}>1 \mathrm{~s}^{-1}$ and $W i>5$. The position of circles formed by particles is not stationary: circles increase the radius and slowly migrate to the periphery of operating cell.

So, large spherical particles arrange as concentric circles in the strong shear flow of viscoelastic polymer matrix. What will happen, if dimensions of particles will be essentially smaller and their shape will be not spherical? To answer this question the same matrix was filled with $7 \%$ wt of clay Cloisite $\mathrm{Na}+$. Clay particles are anisotropic and appear birefringence, i.e., visually detected in polarized light at .rossed polars. This composition demonstrates ordering of particles in strong shear flow $\left(\dot{\gamma}>1 \mathrm{~s}^{-1}\right)$ forming arches and as a limiting stage - circles as well (Fig.11).

Observation in-situ mode allows us to see two kinds of motion: rotational and translational with subsequent formation of ordered circles. The ordering process starts in vicinity of lens apex, but the most regular circles are seen in the middle of vision area, while in periphery of cell where the gap is widening, a tendency only to arrangement of particles is observed. As in previous case, the developed morphology is not stationary - circles "are running" from the center to periphery. It is likely that the driving force of ordering are critical values of shear rate and stress that are maximum near the apex (more precisely at a distance of 0.35 

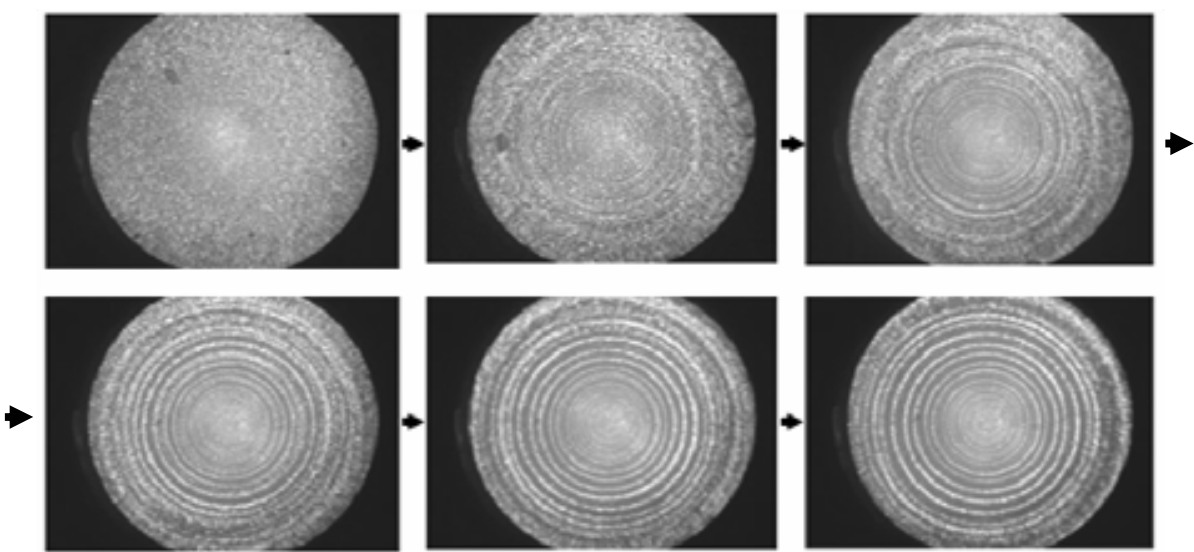

Fig. 11. Subsequent stages of regular morphology formation by $7 \%$ wt of Cloisite $\mathrm{Na}+$ particles in PIB solution. Time interval between frames is $10 \mathrm{~min}$.

$\mathrm{mm}$ from the center at $\mathrm{h} \sim 1 \mathrm{mkm}$ ) and decrease along radius because of non-linear increase of the gap (Fig.12). Movement of circles is likely stipulated by gradient of normal stresses along radius generating driving force acting on a set of particles.

The developed morphology is stable and does not change at least several days. The picture shown in Fig.12 was taken after 24 hours stay the sample after formation of this morphology. It is seen that on a distance more than $4 \mathrm{~mm}$ from the center corresponding to shear rate $\sim 1 \mathrm{~s}^{-1}$, there are no circles. It is critical shear rate corresponding to the definite relation between elastic and viscous forces generating regular morphology formation. One of intrinsic feature of this picture consists in possibility of circle twinning that can be detected for the last circle closed to zone where capability of particles to regular arrangement disappears.

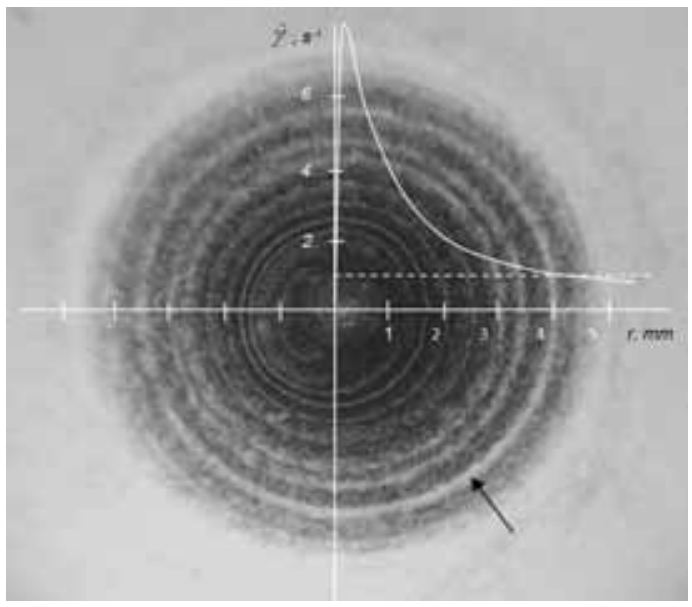

Fig. 12. Micrograph of composite based on PIB solution and Na-montmorillonite particles after 24 hours after circles formation accompanied with dimension scales and distribution diagram of shear rate. Arrow indicates twinning the circle. 
As a starting point of the driving force of regular arrangement of particles in strong shear field, two approaches should be considered. The first one is based on hypothesis about structure transformation of polymer matrix influencing on particles location. The second one - on specific change of rates and stresses fields in presence in viscoelastic matrix of impurities or defects, such as solid particles.

For analysis of the first factor morphological pictures should be added by flow curves of heterophase compositions. As an example, corresponding data for SAN filled with natural and modified Na-montmorillonite are presented in Fig.13. In contrast with classical Einstein law postulating increase of suspension viscosity with volume fraction of solid particles, in our case the viscosity changes with filler content non-monotonically (Fig.13a). The lowest viscosity for systems with Cloisite $\mathrm{Na}+$ is realized for $5 \%$ dispersion. It is strange that this effect is observed for all shear rates used. The effect is not caused by destruction of SAN during mixing procedure because neat polymer was subjected to the same temperatureshear rate-time action, as blends.

In the case of hydrophobic organoclay (Cloisite 20A) evolution of viscosity with filler content looks more natural: the viscosity increases with a filler content at low shear rates (up to appearance of yield stress) and decreases at high shear rates. By the way, similar dependence was obtained before for polypropylene-Cloisite $\mathrm{Na}+$ system (Kulichikhin et al., 2000). It seems to us that a term "Non-Einsteinian viscosity" used in some publications (M.E. Mackay et al., 2003) is not correct because we deal with viscoelastic but not Newtonian medium, particles have anisotropic but not spherical shape, and viscosity measures at different but not at one shear rate. Sooner, there is sense to discuss specific dependence caused by small dimensions of particles, their specific interaction with matrix polymer (Jain et al., 2008) and capability to be oriented in shear stream, by non-natural conformation of macromolecules in absorbed layers, as well as partial intercalation of macromolecules into interspaces of layered aluminsilicates. Difference in behavior of hydrophilic and hydrophobic clays in the same matrix can be explained by specific interaction of nitrile groups with solid surfaces of various polarity and as consequence - by different degree of intercalation. Nevertheless, if to analyze dependences presented in Fig.13b, the decrease of viscosity with a content of clay at high shear rates domain is evident.

Clay particles are rather large in diameter $(\sim 5-7 \mathrm{mkm})$ and small in a thickness $(\sim 20-30$ $\mathrm{nm})$, i.e. they are anisometric and almost plain. Particles of ND are almost spherical, and diameter of individual particle is $\sim 3-5 \mathrm{~nm}$, but they are agglomerated. Traditional mechanical mixing procedure of ND with the same SAN leads to maximum distribution function localized at 1-2 mkm. Nevertheless, it is a filler with isotropic particles and their influence on SAN melt viscosity should be interesting, especially compared with anisometric clay particles.

Dependences of viscosity on shear rate for a set of these compositions are shown in Fig.14. In the field of low shear rates rotational rheometer was used, meanwhile for high shear rates field - capillary apparatus. In the whole range of shear rates (stresses) a decrease of viscosity with filling degree is observed, but viscosity minimum is located for ND content $0.5 \%$ and a scale of viscosity decrease is essentially less than for SAN-natural clay system (only $44 \%$ of SAN viscosity, but not $\sim 6$ times as for previous system).

It should be noted that experiments on capillary viscometer were carried out with capillaries of different $\mathrm{l} / \mathrm{d}$ (at the same diameter). Coincidence of data obtained on different capillaries confirms an absence of sliding. 

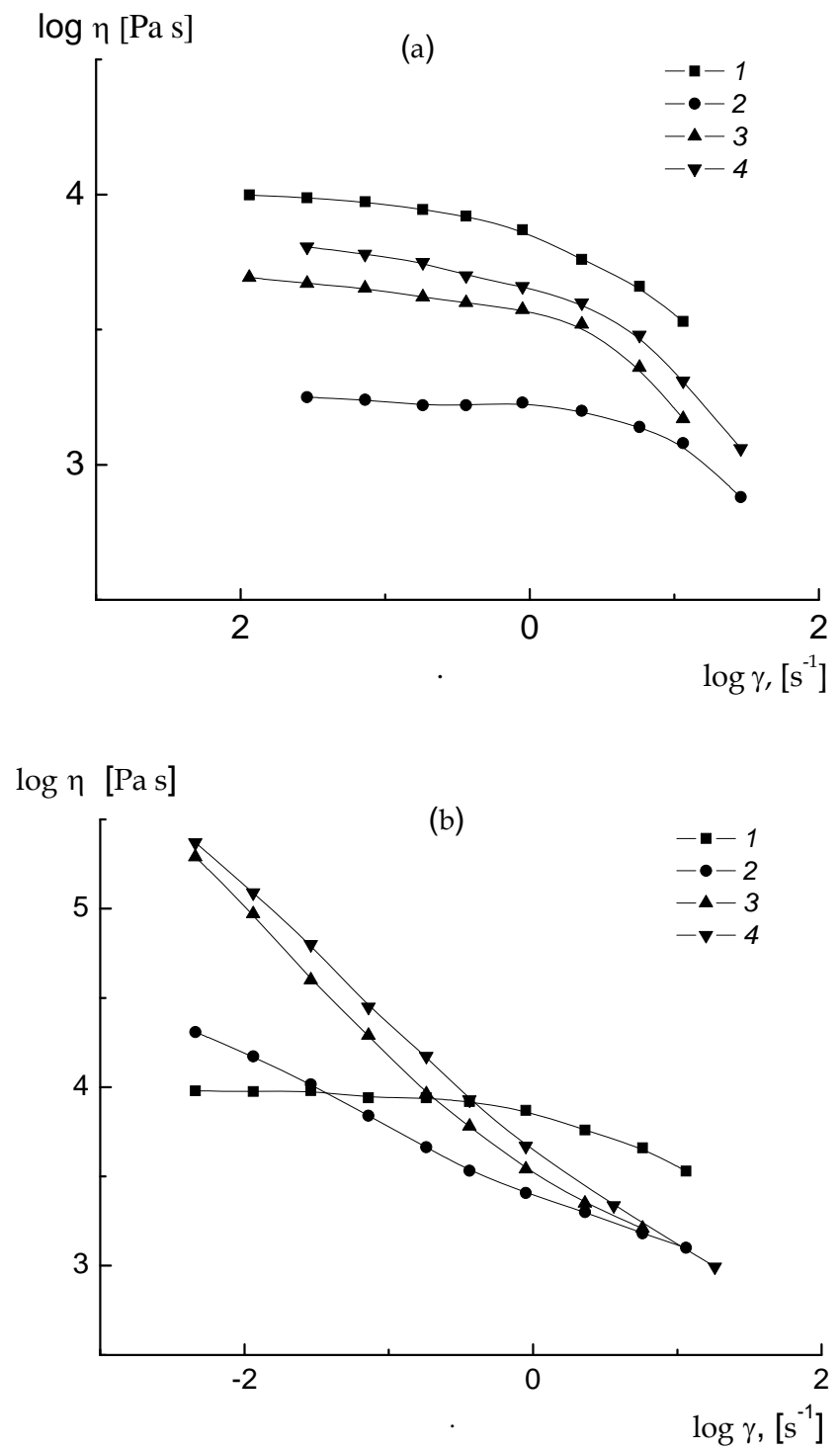

Fig. 13. Dependences of viscosity on shear rate for SAN filled with hydrophilic (a) and hydrophobic clay (b). Content of clay: 0 (1), 5 (2), 10 (3) and 20\% (4). T $=220 \circ \mathrm{C}$.

To understand precisely an influence of particles dimensions on viscosisty, two series of formulations with the same composition steps have been prepared based on PSF matrix and nanodiamonds in traditional and "spurt" regimes. Corresponding dependences of viscosity on ND concentration are presented in Fig.15. For traditional mixing the effect of viscosity decrease at $1 \%$ of ND is prominent, while for compositions prepared via "spurt" regime it is absent, and monotonous increase of viscosity with amount of filler is observed. 


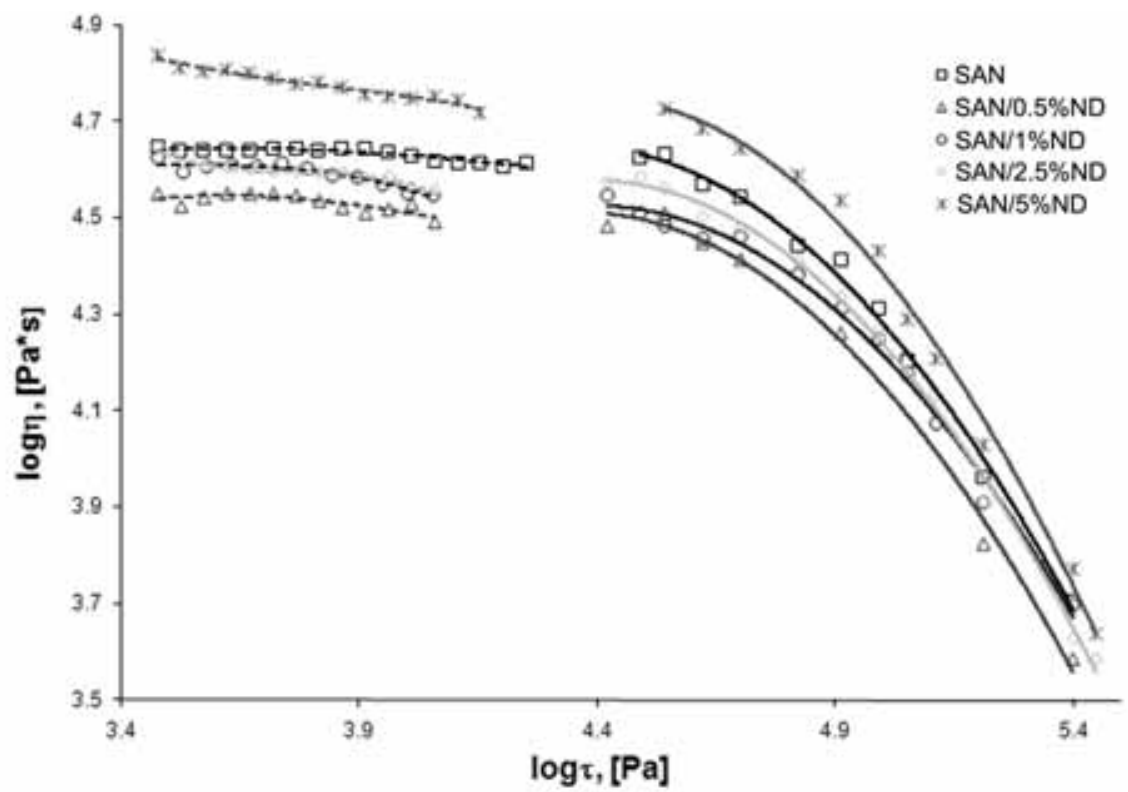

Fig. 14. Dependences of viscosity on shear stress for SAN-ND compositions prepared via reference method. Dotted line - rotation rheometer, solid lines - capillary rheometer. ND content: 0 (1), 0.5 (2), 1.0 (3), 2.5 (4) and 5\% (5).

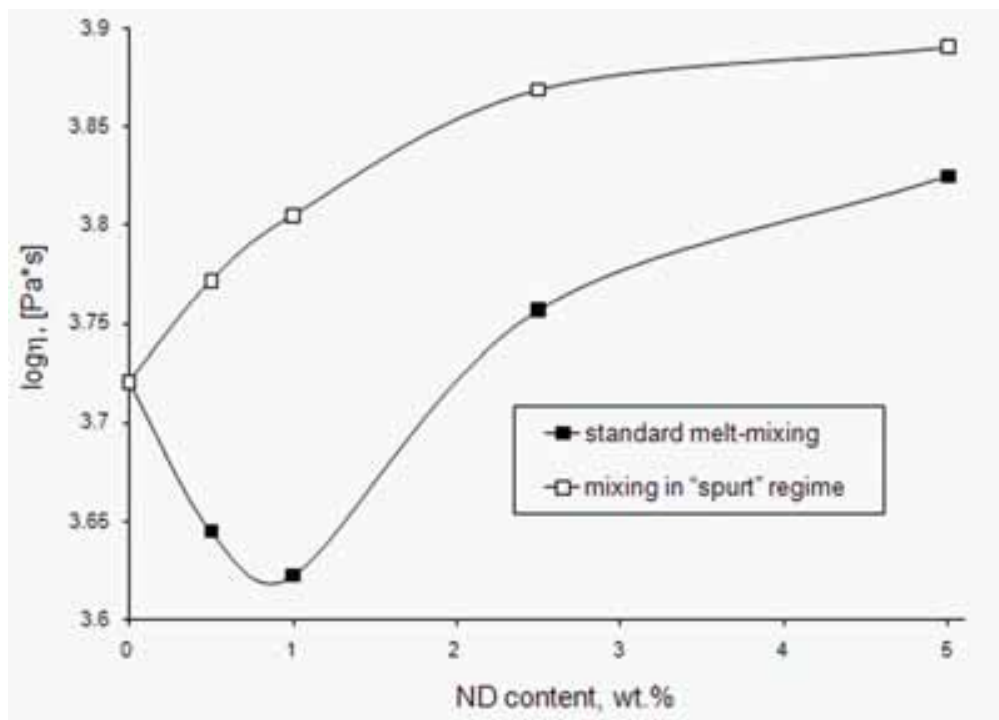

Fig. 15. Concentration dependences of viscosity measured at $\tau=12.5 \mathrm{kPa}$ for PSF-based composites, obtained by two methods. 
Based on a series of experiments with different polymer matrices and fillers, the effect of viscosity decrease at definite content of a filler is not unique inherent for a few pairs polymer-filler only. Presumably, it reflects the common property of filled compositions with viscoelastic polymer matrix. The current tendency consists in more significant scale of this effect for large particles (agglomerates of nanoparticles) and weakening the effect with decrease of particles size. It can disappear as whole for nanosized particles. The effect takes place in the field of strong shear flow, i.e., in the same conditions that inherent for formation of the circle-like morphology. It is likely that both effects are interrelated and we can tell about decrease of viscosity with filling degree for stratified flows.

If a homogeneous melt will be placed in a cell of shear rheometer, in absent of sliding the angular momentum transfers homogeneously from the moving to stationary part (translation mode, Fig.16a). At presence in a melt of anisometric particles capable to rotate in shear field, the second mode of dissipation appears - rotational. At low shear rates superposition of two modes increases the hydrodynamic resistance of a system leading to viscosity growth (Fig.16b). In these conditions a laminarity of stream can be destroyed. However, at high shear rates localization of modes can take place and transition to stratified flow (formation of a circle morphology indicates on reality of this situation) where translation and rotational modes are concentrated in different layers (Fig.16c). We can expect decrease of mechanical energy dissipation and, consequently, decrease of viscosity.

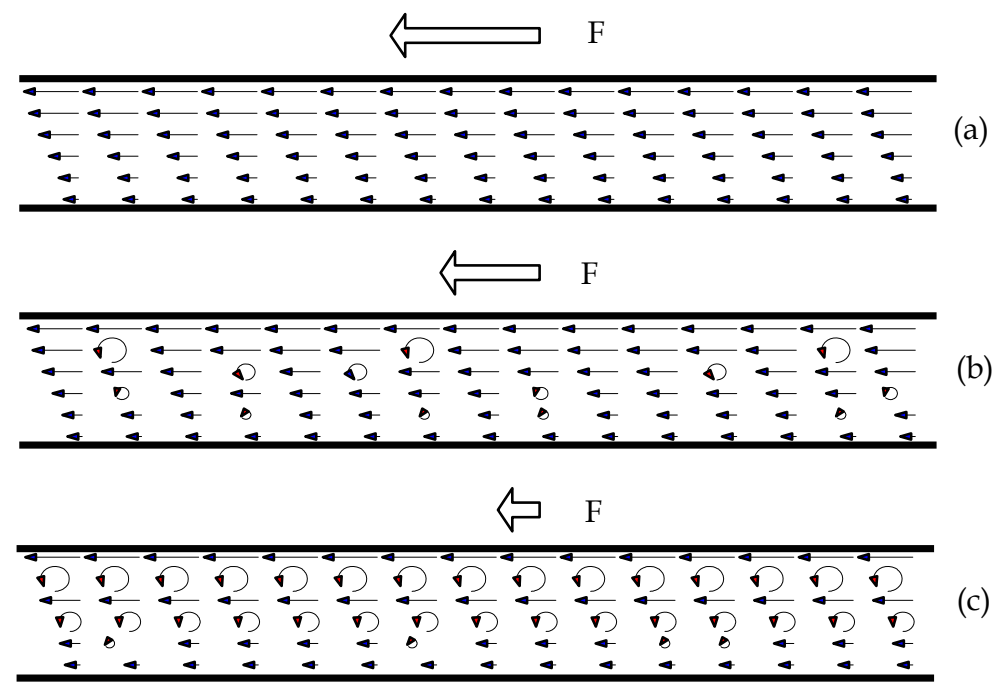

Fig. 16. Scheme of transition from laminar flow of homogeneous system (a) to irregular superposition of translation and rotation modes (b), and to stratified flow (c) in heterogeneous system.

Formation of the circle morphology is typical as well for blends of incompatible polymers (Kulichikhin et al., 2001), and in this case drops of disperse phase should have low viscosity and be enough large (more than critical Taylor radius) to be extended in shear field to form closed circles. The viscosity of two-component blend is a sum of inputs stipulated by viscosity of components and their volume fraction. If we consider the effective viscosity of a homogenous multi-component system then the viscosity is often found to be given by a logarithmic rule of mixing: 


$$
\log (\eta)=\sum_{i} \phi_{i} \log \left(\eta_{i}\right) \text { or } \eta=\prod_{i} \eta^{\phi_{i}}
$$

where the summation runs over all the volume fractions $\left(\phi_{i}\right)$ and viscosities $\left(\eta_{i}\right)$ of the pure components. This sort of expression is commonly used to calculate the viscosity of homogenous polymer blends.

On the other hand, if to assume that a stratified system consisting of $i$ layers exists, we obtain (Abramowitz \& Stegun):

$$
\frac{1}{\eta}=\sum_{i} \frac{\phi_{i}}{\eta_{i}}
$$

It is worth noting that the effective viscosity of the stratified system (eq.9.) is invariably lower than for the homogenous mixture (eq.8.). Thus, if at definite conditions a transition from homogeneous (segmental) to stratified flow takes place, the viscosity should be decreased. As was seen the similar effect of viscosity decrease caused by stratification of stream can be observed at high shear rates for filled systems as well.

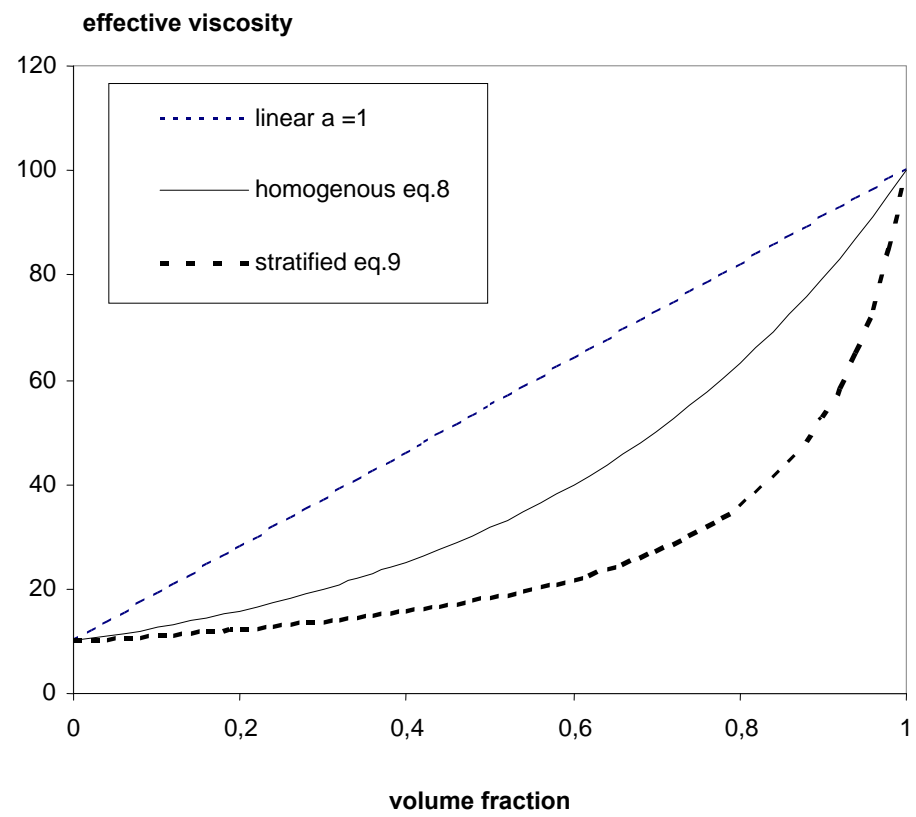

Fig. 17. Linear, geometric mean and harmonic mean viscosity averages for a two component system with viscosities of 10 and 100 (a.u.).

The resulting curves for the various rules of mixing are schematically shown in Fig.17 assuming viscosities of 10 and 100 respectively. When we now apply a fast shear flow on our material the overall dissipation will be higher for the homogenous system than for the stratified system. This means that the flow will act as a driving force to cause the system to phase separate into "high" and "low" viscosity bands. The low viscosity bands then reduce the effective viscosity of the whole system. In the present case of a polymer containing 
nanoparticles the nanoparticles tend to accumulate in the fast flow so that the viscosity increase is effectively reduced via lubrication by the low clay content regions in the flowing system.

So, we have tried to prove that the effect of viscosity decrease can be caused by stratified flow of more-less large particles, that is why it is connected with morphological transformations of a heterophase system. But what is the driving force of regular morphology formation? Earlier, there were suggested two possible reasons: specific change of polymer matrix in strong flow and influence of particles on laminarity of flow. First of all, let us consider, what happens with individual polymer melt in strong flow.

If we will come back to Fig.9, it is possible to select four regions of shear rate corresponding to different rheological responses. Zone I corresponds to Newtonian behavior (the viscosity does not depend on shear rate and shear stresses are much higher than normal stresses). In Zone II non-Newtonian flow starts and this situation can be described as reversible destruction of fluctuation network of entanglements (the viscosity decreases with shear rate and shear stress growth in less extent than shear rate). In Zone II normal stresses become higher than shear stresses (this means that elastic response prevails). At last, Zone IV corresponds to "spurt" behavior (both components of stresses are almost constant).

In the case of capillary flow various kinds of irregularities appear on extrudate surface (Fig.18 ), in the case of rotational flow irregularities of stream (vortexes of elastic turbulence) start on periphery and pass to the center (Fig.19).

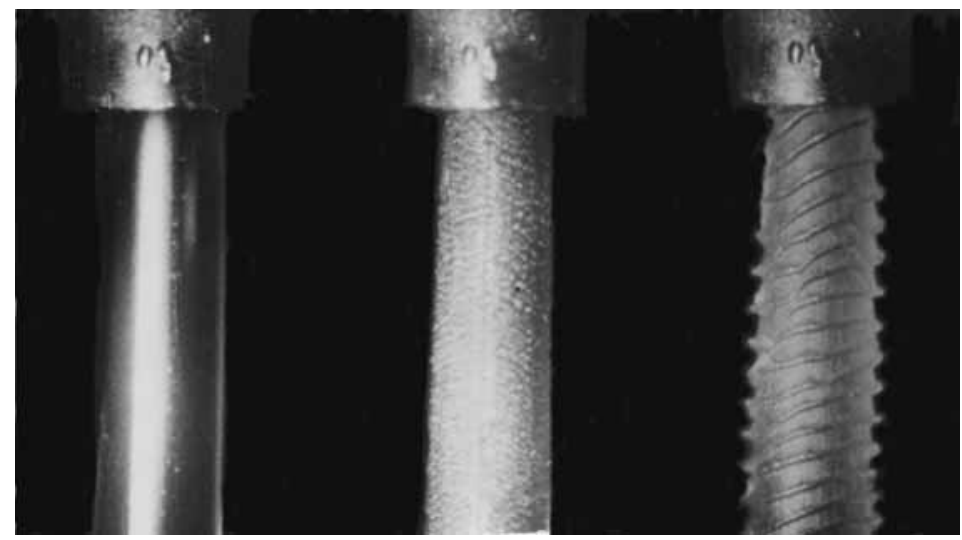

Fig. 18. Irregularities of polymer extrudate surface at increase of shear rate (from left to right).

However, there exists shear stress-shear rate window (pre-"spurt" region) where individual polymer melts appear regular texture. As an example, the surface of cohesion rupture of polycarbonate formed on pre-"spurt" regime realized on both parts after dismounting the operating unit cone and plate is shown in Fig.17. Besides, the new version of "spurt" phenomenon explanation as cohesion rupture but not adhesion slippage should be taken into consideration.

It is surprising, but we come across the circle-like or sooner the spiral texture again. Actually, the sample surface has spiral structure that in a distance looks like concentric circles. Moreover, formation of this surface proceeds via twinning the spiral (see spiral periphery in the bottom part of the picture). 


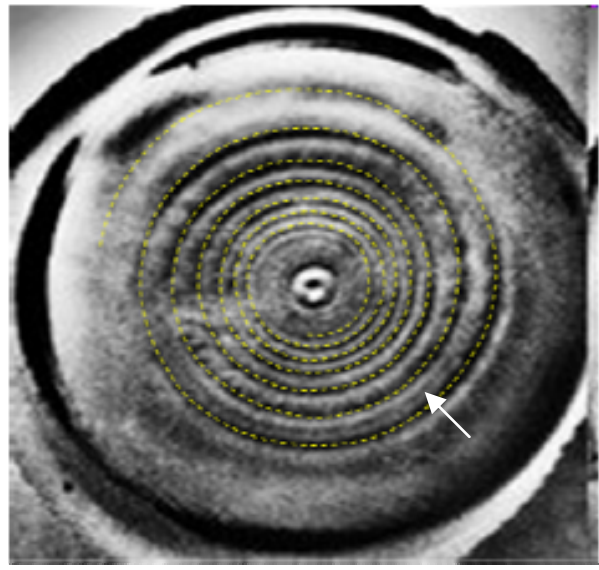

Fig. 19. Irregular surface of polycarbonate in "spurt" regime realized in cone-plate geometry. Dotted line corresponds to "dimple" direction.

Formation of the spiral structure at high shear rates is possible to see directly in transparent cell. The micrograph of PIB melt in transmitting light with a sharp black boundary separating flow zone and the surface formed as a result of medium rupture is shown in Fig.20. The narrow zone of the surface close to flow region has regular spiral structure. Spirals are directed by such a way that they are going from flow region (defectless part in the center of sample). On the earliest stage of the spiral structure formation (Fig.20a) evolution of spirals proceeds via twinning of their period (arrows show doubling of furrows). With time (Fig.20b) spiral ridges transform to ring-like morphology.
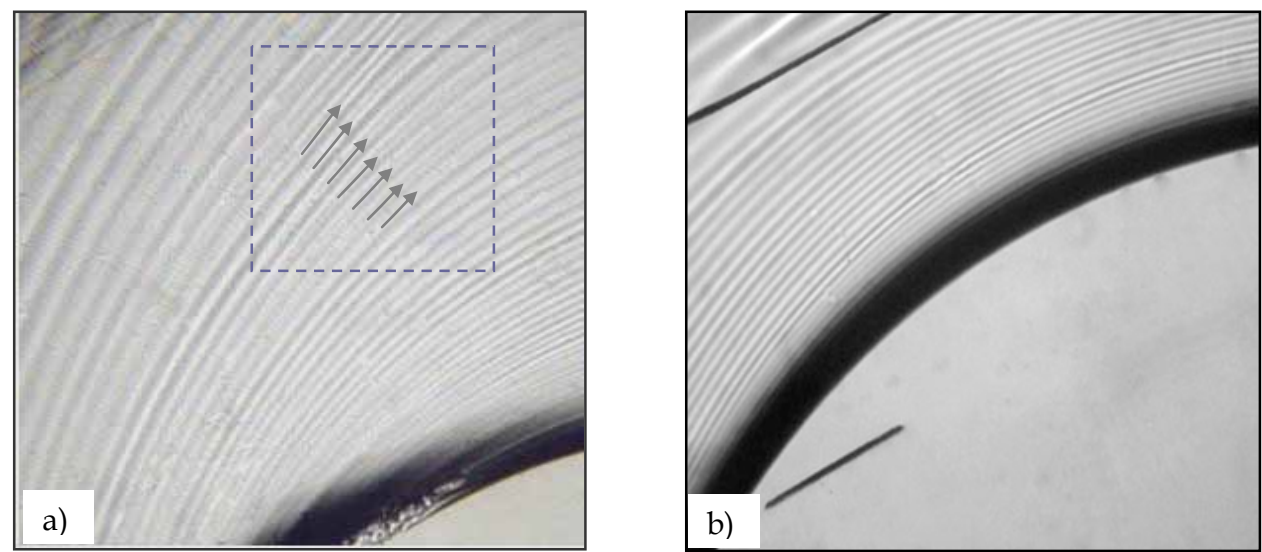

Fig. 20. Subsequent stages of texture formation from spiral (a) to circular (b) for PIB melt. The distance between lines in figure $b$ ) is $1 \mathrm{~mm}$.

To know what happens in the flow zone the optical interferometry was used. The interference pattern of the flow zone for polybutene melt in vicinity of lens pole is shown in Fig.21a. The regular texture becomes visible at angular velocity of a plate of the order of unit of rad/s as alternating light and dark bands spreading from the center to the periphery. In transmitting non-monocromatic light this zone remains transparent. 


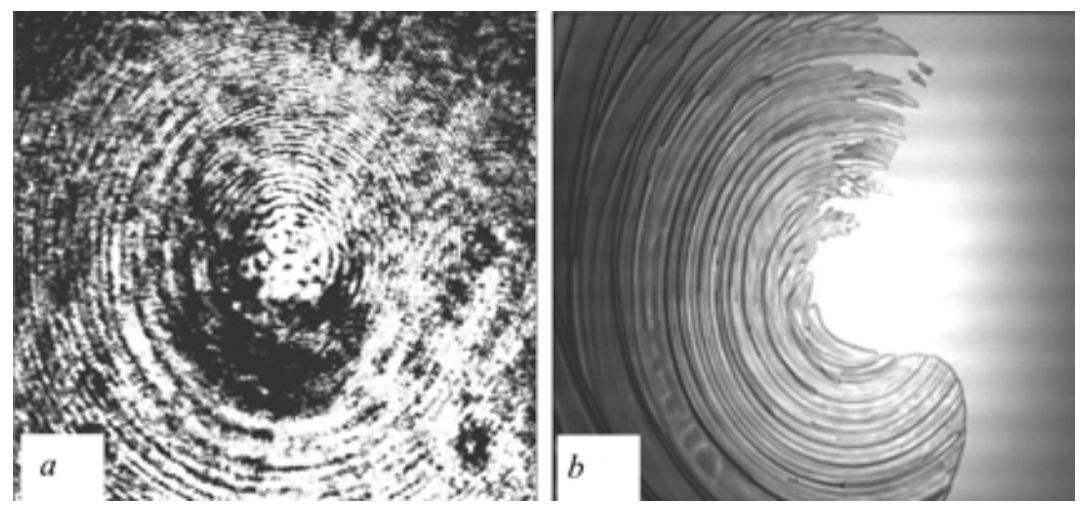

Fig. 21. Texture of polybutene in interference (a) and white light mode (b) in vicinity of rotation axis of the lens-plate cell at $\omega=0.5 \mathrm{rad} / \mathrm{s} \mathrm{(a)} \mathrm{and} 1 \mathrm{rad} / \mathrm{s} \mathrm{(b).}$

Comparison of Figs. 21 and 20, allows us to judge that spiral texture of the surface breakage on a periphery of stream is continuation of texture initiating in "transparent" zone (transmitting white light). Interference spiral structure appears as a result of modulation of refraction index due to photoelastic effect. At high shear rates $(\mathrm{Wi}>1)$ in polymer melt elastic stresses (reversible strains) appear that are visualized by optical interferometry. Dark fields correspond to extension, light ones - to compressive stresses. The dark "half-moon" region in Fig.21a corresponds to initiation of "spurt" or cohesion rupture of a melt. At increase of angular velocity up to $1 \mathrm{rad} / \mathrm{s}$ the regular instabilities appear visible in white light (Fig.21b). Effects described above are observed in strong flow where elasticity of polymer matrix becomes a dominant factor of its rheological behavior. The same time it is well-known appearance of instability or secondary flows may take place in viscous inelastic liquids (classical example is so-called Tailor vortexes forming in confined geometry as a result of inertial effects. What is a difference between inertial and elastic instability? We were lucky to observe a transition from one kind of instability to another one for very similar systems varied with different level of elasticity.

At shear flow of diluted aqueous dispersion of nanodiamonds in presence of non-ionic surfactant (Tween 80) the initially homogeneous dispersion is separating on a system of rotating radial formations formed by ND particles (Fig.22). These figures are very similar to Tailor vortexes (Larson, 1991), when inertial forces become to be dominant over viscous forces. Formation of vortexes starts in vicinity of the lens pole and they develop to periphery of stream that is similar to circles evolution considered above. The main distinction consists in direction of instability figures: longitudinally or transversally to shear.

One can see the transition from inertial instability to elastic one, if $1 \%$ of HPC will be introduced to aqueous medium of the same dispersion. The rather weak elasticity is enough for drastic change of stream morphology: instead radial structures concentric circles or spirals are forming. (Fig.23).

Thus, experiments carried out as with nanocomposite precursors, as with dispersions in Newtonian or weakly viscoelastic media, as with individual polymer melts indicate on formation of regular structures in strong shear flows. The main reason of regular structures development should be the intrinsic profile of elastic stresses and strains, i.e., their periodic modulation. That is why the profile of elastic forces in polymer melts and the texture of particles distribution in strong flows should be similar. This circumstance is decisive in the search of driving forces of ordering. 

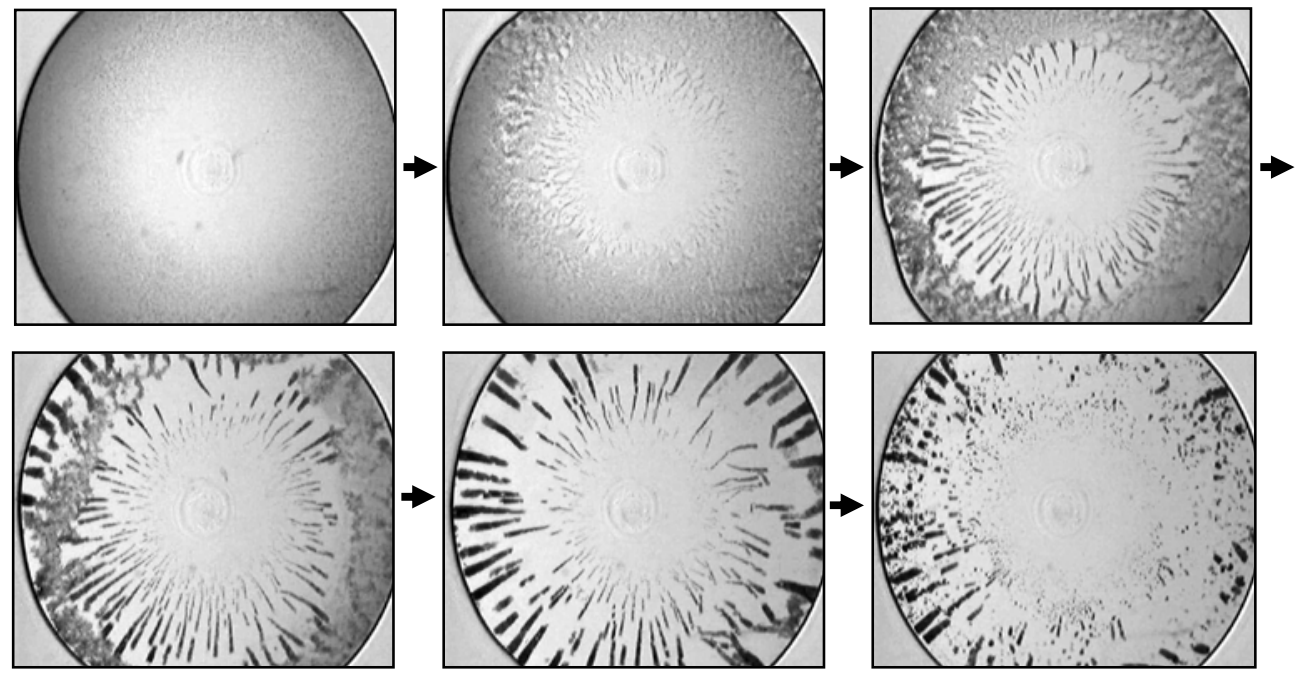

Fig. 22. Morphology evolution with strain in aqueous suspension of ND in presence of surfactant.

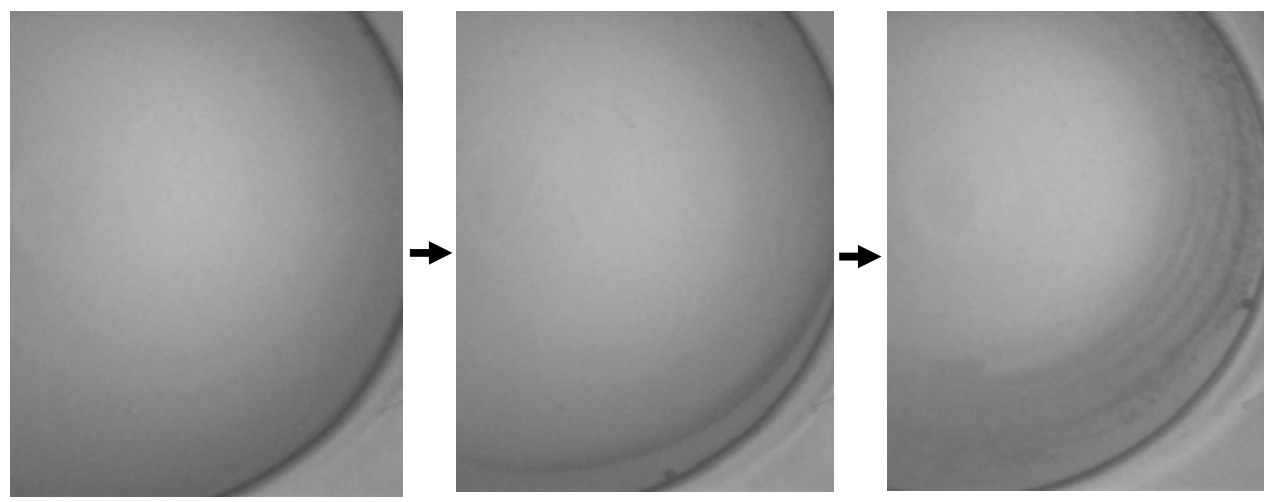

Fig. 23. Morphology evolution with strain in suspension of ND in $1 \%$ aqueous solution of HPC.

It is likely that the main factor of regular arrangement of particles is action on them of elastic forces from polymer matrix that itself becomes structured or textured in strong flows. Filler particles play a role of tracers reflecting stratified polymer flow.

\subsection{Rheo-X-ray data}

For complex liquids, such as liquid crystals, colloid systems, incompatible polymer blends, nanocomposite precursors combination of rheological testing with structural methods is a stable tendency now. Strong influence of shear rates and shear stresses distribution in intense shear on stream morphology has forced us to look more detail on structure transformations on a level of crystalline lattices. Together with Dutch partners (Makarova et al., 2010) a series of experiments was carried out at the Delft University of Technology 
where a combination of Couette cell and X-ray scattering cell was developed. Since the most fruitful results should be obtained on structure active objects, the aqueous LC solution of HPC was used as a matrix and crystalline Na-montmorillonite as a filler.

For choice of HPC solution concentration, the phase diagram of HPC-water system was constructed by means of optical microinterference method (Malkin et al., 1983); Makarova, 2007). According to this method, interacting substances are placed in side-by-side manner in the wedge gap and the diffusion profile is registered as evolution of interference fridges inherent to each component. In equilibrium state the terminal concentrations are taken as limits of reagents solubilities at different temperatures and used for constructing the phase diagram.

As an example, the interference patterns at two temperatures are presented in Fig 24a. Along the diffusion zone at $29^{\circ} \mathrm{C}$ four different sections are observed that correspond to the traditional phase sequence (from left to right) inherent to solutions of stiff-chain polymers: isotropic (I), biphasic (II), LC (III) and CS (IV), as well as the exact location of boundaries between them. With increase of temperature the interference pattern changes dramatically. Sections I and II disappear and the sharp border identified as amorphous phase separation appears. Based on these data, the phase diagram of HPC-water system was drawn (Fig. 24b) (Kulichikhin et al., 2010), reflecting superposition of two kinds of phase equilibria: amorphous (binodal with low critical mixing temperature) and LC equilibrium, theoretically predicted in (Flory, 1956). With increase of polymer concentration isotropic solution (I) transforms to liquid crystalline (LC), and this transition proceeds through biphasic region of coexisting of I and LC phases. At extremely high content of HPC formation of crystal solvate (CS) phase is possible. Of course, the most interesting solutions as matrices for rheo-X-ray study are anisotropic, and to make the final choice the concentration dependence of viscosity was considered.

As a rule, the viscosity decreases with appearance of LC-phase due to change of flow mechanism from segmental movement to displacement of domains which easily orient in shear field (Kwolek, 1972; Papkov et al., 1974). The same situation takes place for HPC solutions. The corresponding dependence of viscosity on HPC content is shown in Fig 25.

As is seen, the viscosity minimum appears at HPC content of $50 \%$ corresponding to biphasic region. This concentration was chosen as a matrix for dispersion with clay. By the way, presence of clay does not influence the location of equilibrium lines of the phase diagram, but leads to increase of viscosity at low shear rates almost proportionally for whole concentration diapason.

Before analysis of difractograms at flow, we need to know features of X-ray scattering of neat HPC and clay powders. These data are shown in Fig.26.

For HPC scattering maxima at $2 \Theta=8.8$ and $20.2^{0}$ are inherent. Unfortunately, main reflexes of clay are located in vicinity of these angles: at 6.8 and $19.7^{0}$. The first one is the basal reflex corresponding to interspace distance in layered crystalline structure. It changes the position depending on intercalation (penetration) of any species into clay structure. Partially, its position depends on water content shifting with increase of moisture level to smaller angles. That is why we can expect change of basal reflection location for aqueous solutions.

First of all, the neat solution was suffered to shear in Couette cell with different shear rates with registration of difractogram. Corresponding results are shown in Fig. 27. HPC orientation is expressed as a strong reflex on meridian of difractogram at $2 \Theta=6.1^{0}$ and becomes prominent at shear rate higher than $\sim 20 \mathrm{~s}^{-1}$. 


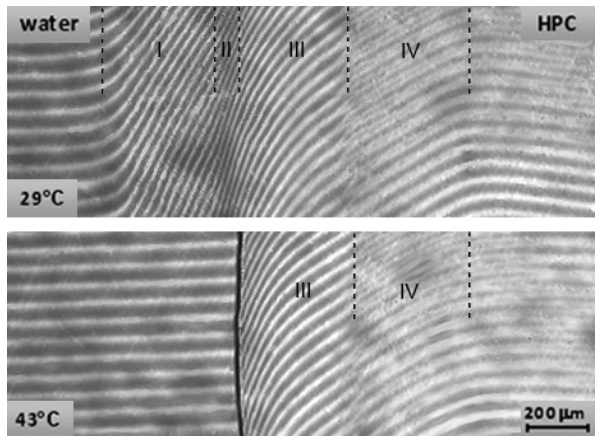

a)

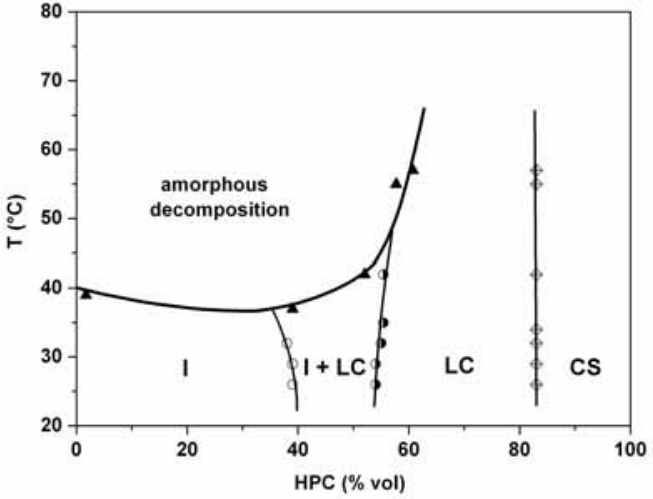

b)

Fig. 24. Microinterference patterns of interdiffusion zone of HPC-water system at two temperatures (a), and phase diagram based on these data (b).

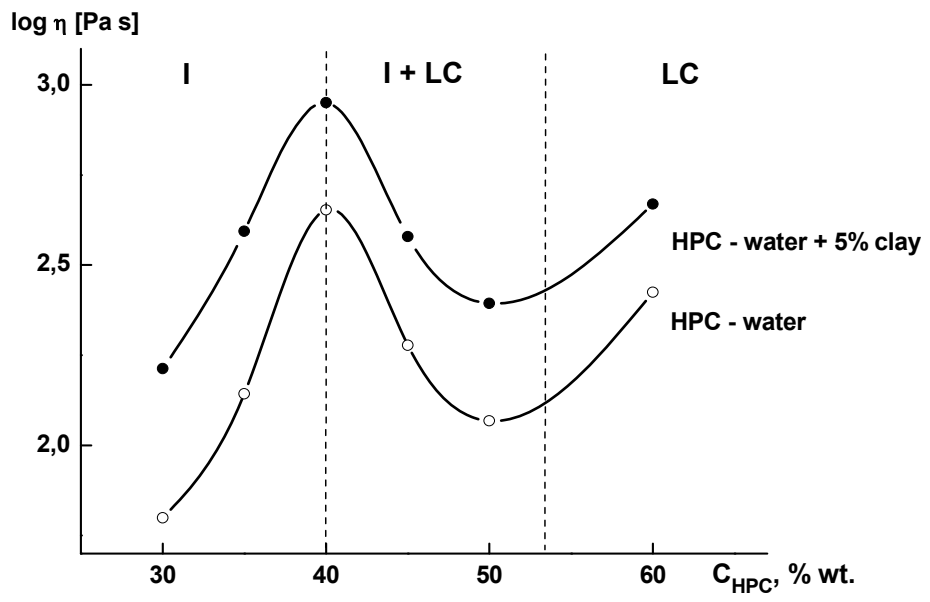

Fig. 25. Concentration dependence of viscosity for HPC solutions and composites on their base measured at shear rate $0.25 \mathrm{~s}^{-1}$ and ambient temperature. 


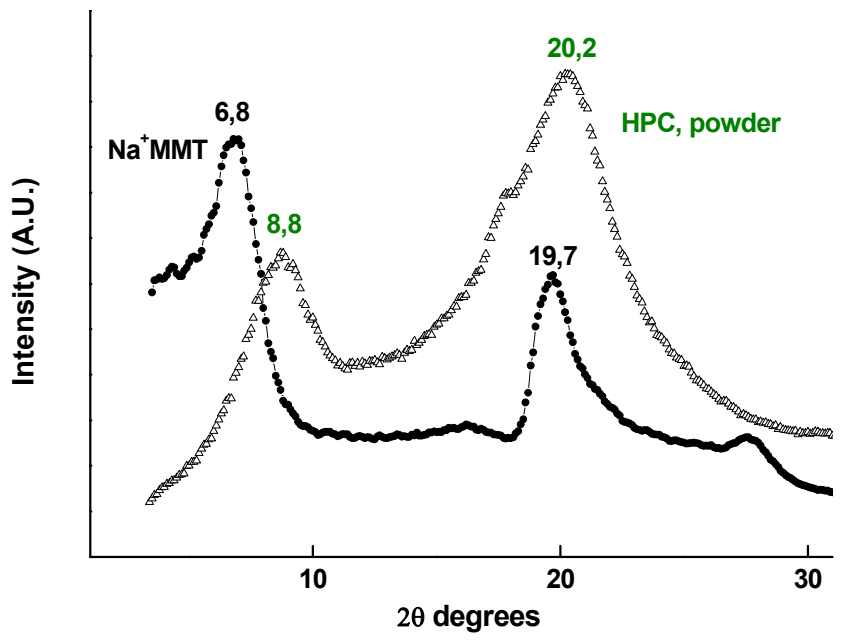

Fig. 26. Diffractograms of Na-montmorillonite and HPC powders.

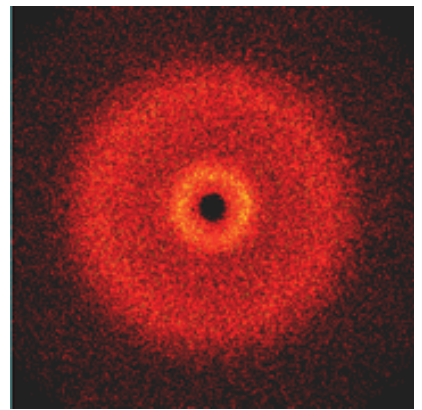

a)

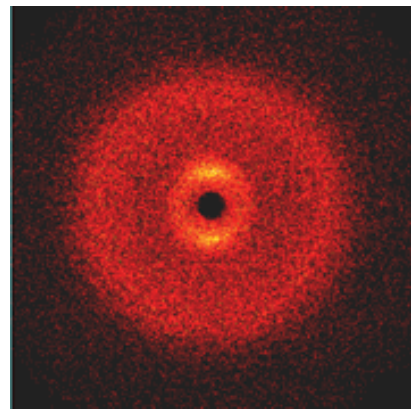

b)

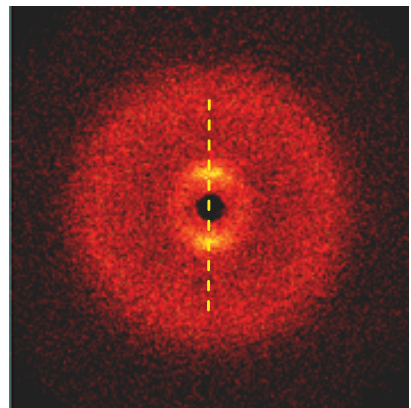

c)

Fig. 27. 2-D diffraction patterns of 50\% HPC solution exhibiting the dependence of orientation on shear rate at $0(\mathrm{a}), 28.2(\mathrm{~b})$ and $226.1 \mathrm{~s}^{-1}(\mathrm{c})$.

Distribution of intensity in azimuthal angles (Fig.28) show that weak initial orientation caused by loading of solution into the gap between two capillaries at flow rotates on $90^{\circ}$, i.e., LC solution becomes oriented along the shear direction.

Dependence of order parameter calculated on shear rate is shown in Fig.29. The initial value of $\left\langle\bar{P}_{2}\right\rangle(0.16)$ is stipulated by orientation at loading and under action of shear its value increases to 0.66 .

In the case of nanocomposite precursor we need to separate reflexes inherent to HPC and clay. Looking at difractograms in $2 \Theta$ angles (Fig. 30), one can see that basal clay reflex in aqueous solution is shifted to $\sim 3.2^{0}$, while the reference HPC reflex remains at $6.6^{0}$, so, it is easy to distinct them. In the rest state the HPC reflex is rather weak and this means that major fraction of HPC molecules is connected with clay due to absorption and intercalation. 


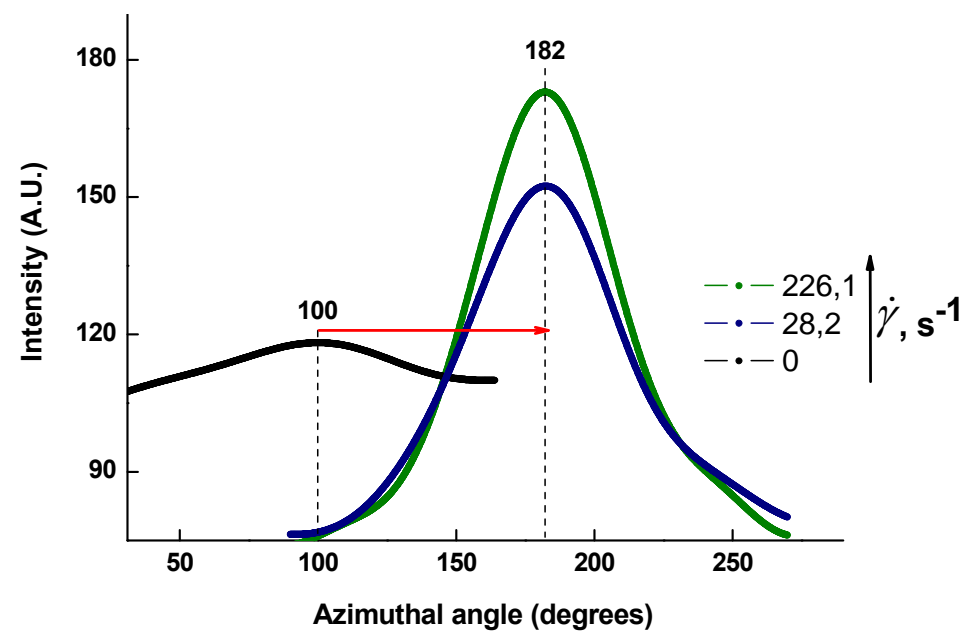

Fig. 28. Distribution of azimuthal intensity of the strongest reflex of $50 \%$ HPC solution at different shear rates.

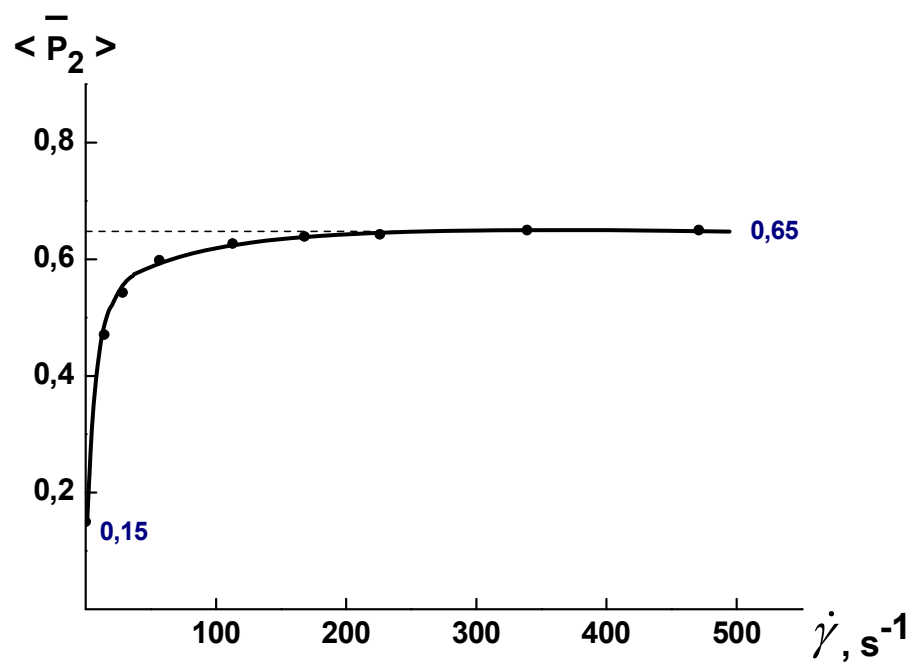

Fig. 29. Dependence of order parameter on shear rate for $50 \%$ HPC solution.

With increase of shear rate the redistribution of intensities between reflections inherent to clay and HPC occurs, and this process can be caused by release of HPC molecules from clay force field. The microphase separation takes place. Details of structure change might be understood from analysis of X-ray patterns of $50 \%$ HPC solution filled with $5 \%$ of Namontmorillonite presented in Fig. 31.

As is seen, in initial state "the loading effect" leads to superposition of reflexes inherent for HPC and clay on equator of difractogram. With increase of shear rate these reflexes become separated: the HPC reflex moves on meridian, and clay reflex remains on equator. These displacements are expressed clearly on difractograms in azimuthal angles (Fig. 32). 


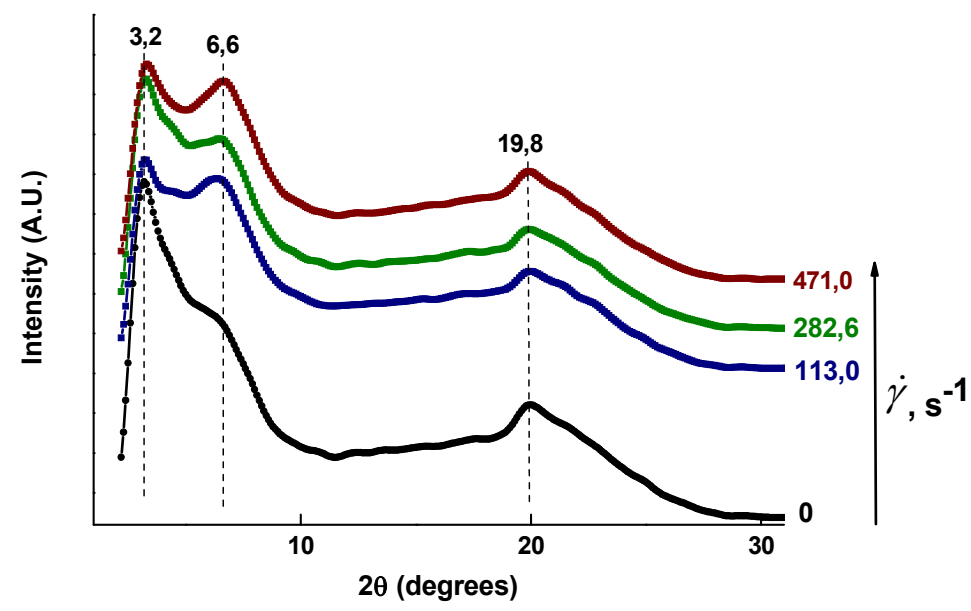

Fig. 30. Evolution of diffractograms along $2 \Theta$ angle for $50 \%$ HPC solution containing $5 \%$ of Na-montmorillonite with shear rate.

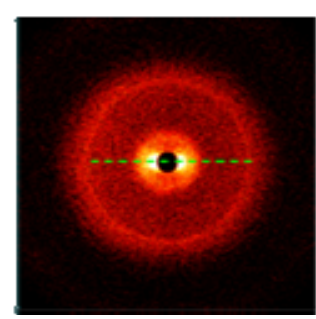

a)

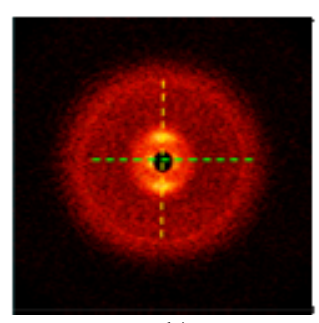

b)

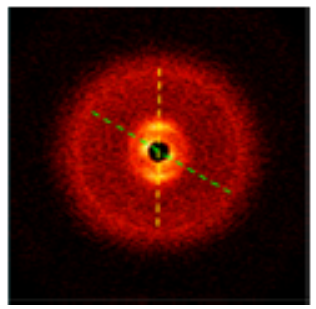

c)

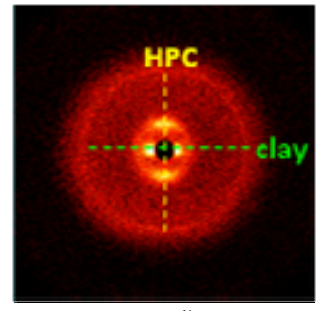

d)

Fig. 31. 2-D diffraction patterns of 50\% HPC solution filled with $5 \%$ of Na-montmorillonite on shear rate equal to: 0 (a), 113.0 (b), 282.6 (c) and $471.0 \mathrm{~s}^{-1}(\mathrm{~d})$.

After engaging "equilibrium positions", evolution of orientation for each component proceeds in different way. For HPC already at low shear rate the orientation angle becomes $180^{\circ}$ but its intensity changes with increase of shear rate non-monotonically. The maximum orientation for clay is observed after loading, while the beginning of flow leads to decrease of basal reflex intensity. With increase of shear rate this reflex may oscillate between 245 and $270^{\circ}$, but its intensity increases monotonously.

Dependences of order parameters (separately for HPC and clay) on shear rate (Fig. 33) indicate on existence of region of critical shear rates (around $300 \mathrm{~s}^{-1}$ ) where both parameters suffer deviation from smooth evolution. For HPC in this region the order parameter demonstrates wide minimum, and for clay the value of order parameter becomes dependent on flow time (or total strain). In the first moment after flow starts up it decreases sharply, but with increase of strain it comes back to high values. This feature led to necessity of detail research of X-ray patterns evolution in time at highest shear rate of $471 \mathrm{~s}^{-1}$ to understand the strength of developed structure. These results are presented in Fig. 34.

As is seen, at deformation time between 70 and 75 min the clay reflex changes the position and moves from equator to meridian. At higher observation time this situation does not 


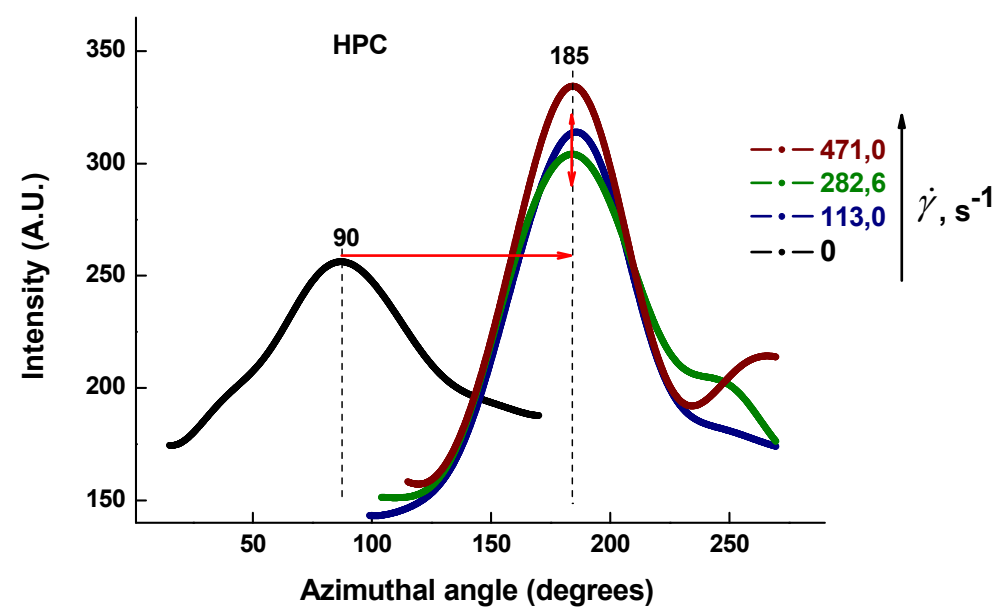

a)

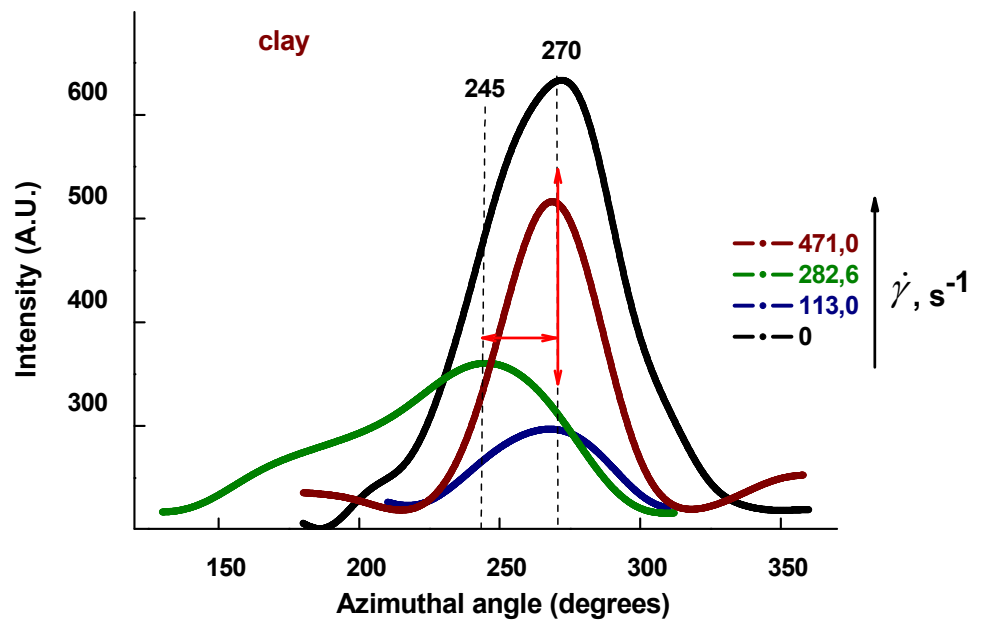

b)

Fig. 32. Distribution of azimuthal intensity of 50\% HPC solution filled with $5 \%$ of Namontmorillonite at different shear rates for HPC and clay separately.

change. Therefore, at strain of the order of $2.10^{6}$ the catastrophic transformation of clay particles orientation takes place. This phenomenon is reflected on the dependence of order parameter on time (Fig. 35) that jumps down in this region.

If to look on evolution of difractograms in $2 \Theta$ angles, it is evident the redistribution of intensities between reflexes 6.6 (HPC) and 3.2० (clay): the first one decreases in favor of the second one (Fig. 36). Moreover, the last reflex moves to position of 3.10. This situation is opposite to observed evolution of difractograms with shear rate (Fig. 30) where intensity of the $6.6^{\circ}$ reflection increases and $3.2^{\circ}$ decreases. This means that clay structure suffers drastic transformation at definite strain. What kind of is this transformation? 


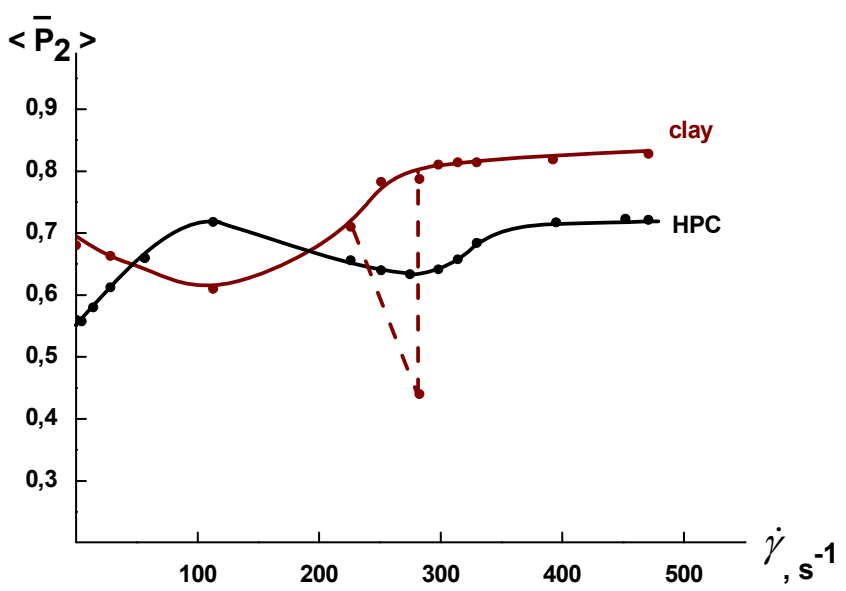

Fig. 33. Evolution of the orientation of HPC and clay with shear rate for $50 \%$ HPC solution filled with $5 \%$ of Na-montmorillonite.

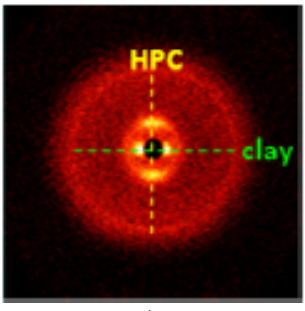

a)

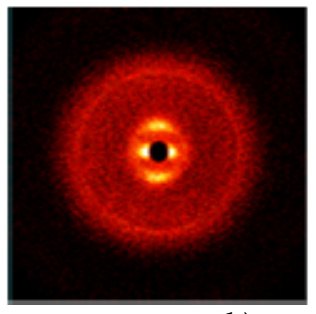

b)

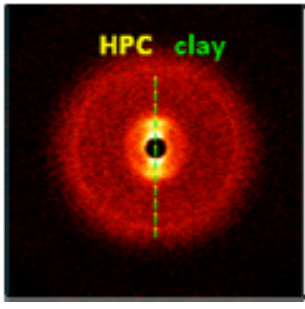

c)

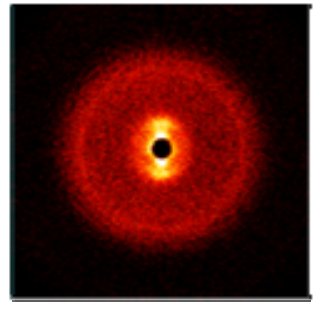

d)

Fig. 34. 2-D diffraction patterns of 50\% HPC solution filled with $5 \%$ of Na-montmorillonite at 5 (a), 70 (b), 75 (c) and $115 \mathrm{~min}(\mathrm{~d})$ after reaching the shear rate of $471 \mathrm{~s}^{-1}$.

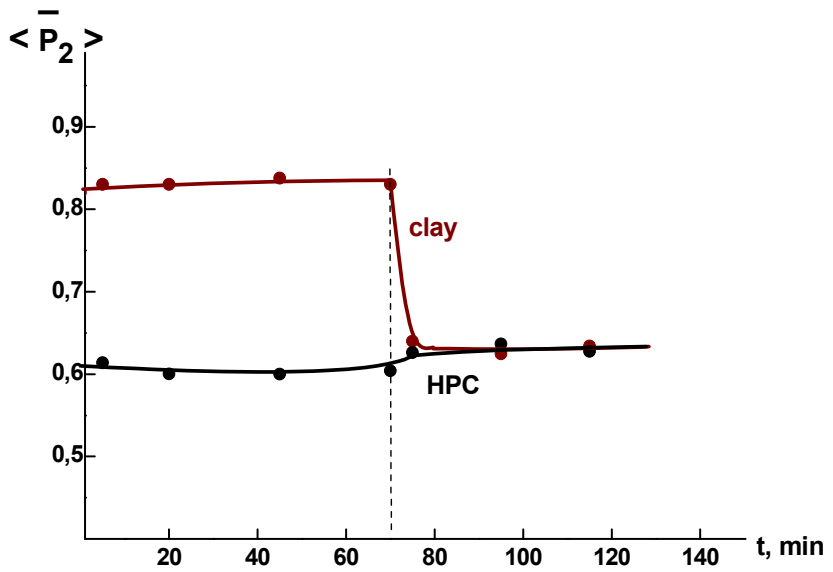

Fig. 35. Dependences of order parameter of HPC and clay for suspension under consideration at prolonged deformation at shear rate of $471 \mathrm{~s}^{-1}$. 


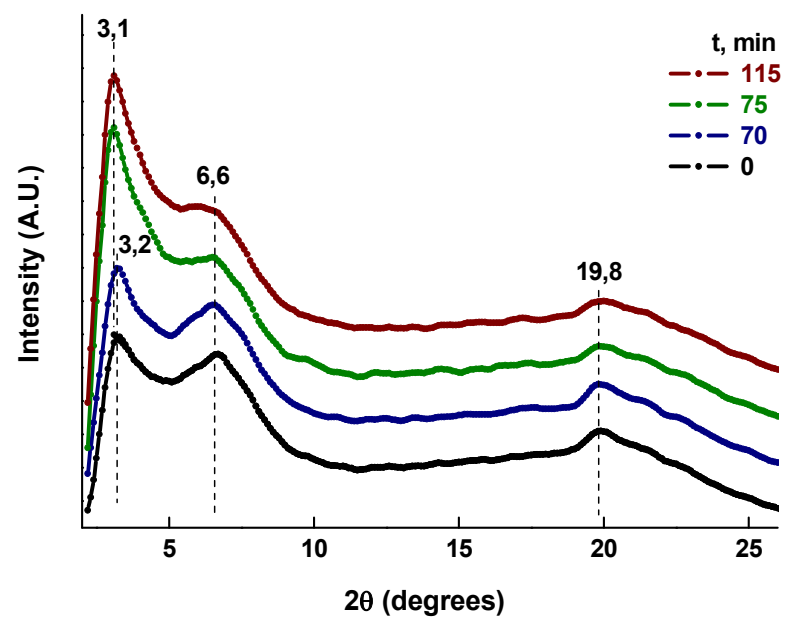

Fig. 36. Diffractograms of 50\% HPC solution filled with $5 \%$ of Na-montmorillonite at prolonged deformation at $471 \mathrm{~s}^{-1}$.

First of all, let us accept that so strong clay reflex (in all versions of our experiments) reflects macro-ordering of clay platelets. This means that they form extended formations similar to chains. In the case when clay reflection is located in equator it should be long sequences oriented by axis perpendicular to platelet thickness along the shear direction. Such situation is very similar to columnar mesophase that looks like stacks of coins. This structure becomes stable at shear rates corresponding to circle-like morphology formation described above and observed in transparent unit (see comparison of hypothetic arrangement of particles in cell Couette and in cone-plate unit in Fig.37). The concurrence of two kinds of mesophases can lead to smooth decrease of HPC orientation and to dependence of clay orientation on time (pronounced decrease of orientation on start-up stage) evident from Fig.33.
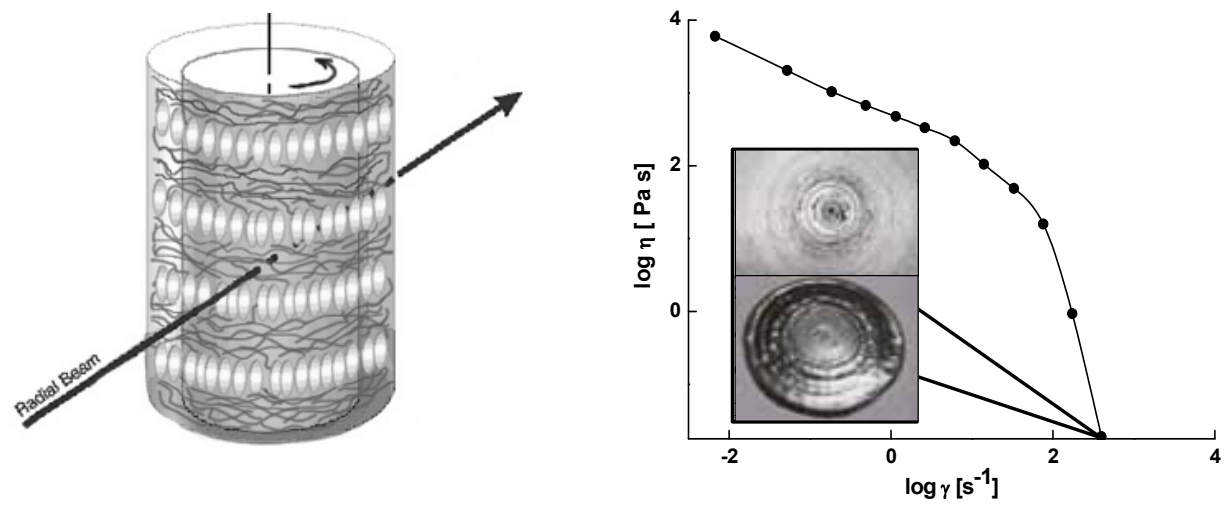

Fig. 37. Hypothetic arrangement of particles in Couette cell (a) and in cone-plate unit (b). As is seen from dependence of viscosity on shear rate regular ordering proceeds at shear rate of $\sim 300 \mathrm{~s}^{-1}$. 
After prolonged strain at high shear rate the clay reflex is turned on $90^{\circ}$ and displaced from equator to meridian, the columnar structure should be destroyed as a result of flow instability and accumulation of heat due to viscous friction, and probably be converted to the version of discotic mesophase (Schmidt et al., 2000) with orientation of the axis transversal to platelet thickness along the vorticity axis (in our case it coincides with the long axis of capillaries forming the Couette cell). This situation is drawn schematically in Fig. 38. Clay particles become less oriented and their order parameter decreases sharply at this time.

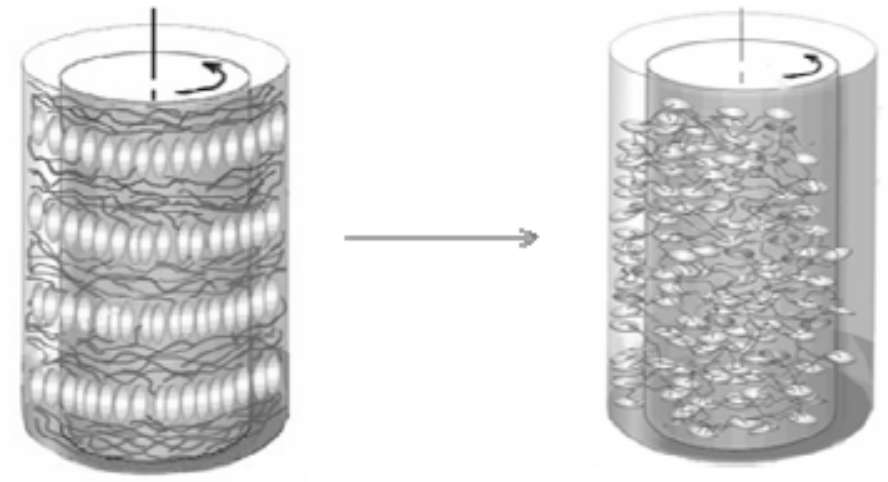

Fig. 38. Scheme of probable transformation of columnar structure to discotic one.

Thus, morphological and structural data are interrelated. In strong shear flow when reversible deformations become dominant something happens with a system leading to regular arrangement of clay particles as on optical level, as on a level of crystalline cell. What does it mean "something", how to touch the possible driving forces of regular ordering?

We will not touch the theoretical background here in details, since the paper is experimental that is more important now in the field of nanocomposites area. But the analysis of literature and our own data suggest that high deformation rates in flow of viscoelastic micellar colloid and polymer systems lead to the flow-to-rubber-like transition. It is the limit of the strong non-linear behavior of elastic liquids. As a result, instability and different effects demonstrating formation of ordered structures were observed. The conception describing the behavior of such a kind based on the refusal from continuum field approach and developing a discrete model which consists in elastic rubber-like grains is proposed and discussed in (Semakov \& Kulichikhin). This is a model of behavior of an elastic liquid in the strong non-linear region of shear rates and stresses. The behavior of the structure elements in this model is determined by their elastic deformations in shear flows (transition from a spherical to ellipsoidal shape of grains and their orientation) and by elastic interaction of these elements due to their collisions. The computer simulation demonstrated that this model really describes the consequence of elastic instability as the chaos-to-order transition and creation of experimentally observed regular structures. This is rather rare case when investigation of nanocomposite gave a food for the new vision of polymer behavior at intense shear strains in spite of a role of particles not more than tracers detecting and contrasting the texture of polymer matrix.

From position of processing conditions of nanocomposites based on rheological principles, it is more important to consider in short mechanical properties reached by declared in this Chapter approaches. 


\subsection{Mechanical properties}

The best tensile properties were achieved for composites obtained by melt-mixing in "spurt" regime. Maximal values of SAN, PSF (neat polymers were treated in the conditions of composites preparing) and the nanocomposites are presented at Table 1.

\begin{tabular}{|l|c|c|c|}
\hline Parameter & Polymer matrix & ND content, wt.\% & Value \\
\hline \multirow{3}{*}{$\begin{array}{l}\text { Young modulus, } \\
\text { G max }\end{array}$} & \multirow{2}{*}{ SAN } & 0 & 2.12 \\
\cline { 3 - 4 } & & 2.5 & 2.95 \\
\cline { 2 - 4 } & \multirow{2}{*}{ PSF } & 0 & 1 \\
\hline \multirow{3}{*}{$\begin{array}{l}\text { Tensile strength, } \\
\sigma_{\max }, \mathrm{MPa}\end{array}$} & \multirow{2}{*}{$\mathrm{SAN}$} & 5 & 1.9 \\
\cline { 3 - 4 } & & 0 & 46 \\
\hline \multirow{3}{*}{ Elongation at break, $\varepsilon, \%$} & PSF & 0.5 & 65 \\
\cline { 3 - 4 } & \multirow{2}{*}{$\mathrm{SAN}$} & 0.25 & 103 \\
\cline { 3 - 4 } & \multirow{2}{*}{ PSF } & 0 & 3 \\
\cline { 3 - 4 } & & 0.5 & 3.6 \\
\cline { 3 - 4 } & & 0 & 32 \\
\hline
\end{tabular}

Table 1. Tensile properties of polymer-ND composites obtained by melt-mixing in "spurt" regime.

The basic tensile properties of the composites were improved significantly. The optimal filler content is $0.5 \%$ by weight. Increases in Young modulus and strength indicate that nanodiamonds are acting as reinforcing agents in the polymer matrix by homogeneous redistribution of load between bulk polymer and absorbed layers around ND particles. The decreasing of mechanical characteristics of the composites obtained by a standard meltmixing, observed in several papers including this one, can be explained by presence of a considerable quantity of the large agglomerates acting as a defects of the system.

Composites PSF/ND has been tested for impact strength. Fig.39 shows the influence of ND concentration and method of composite fabrication on impact strength.

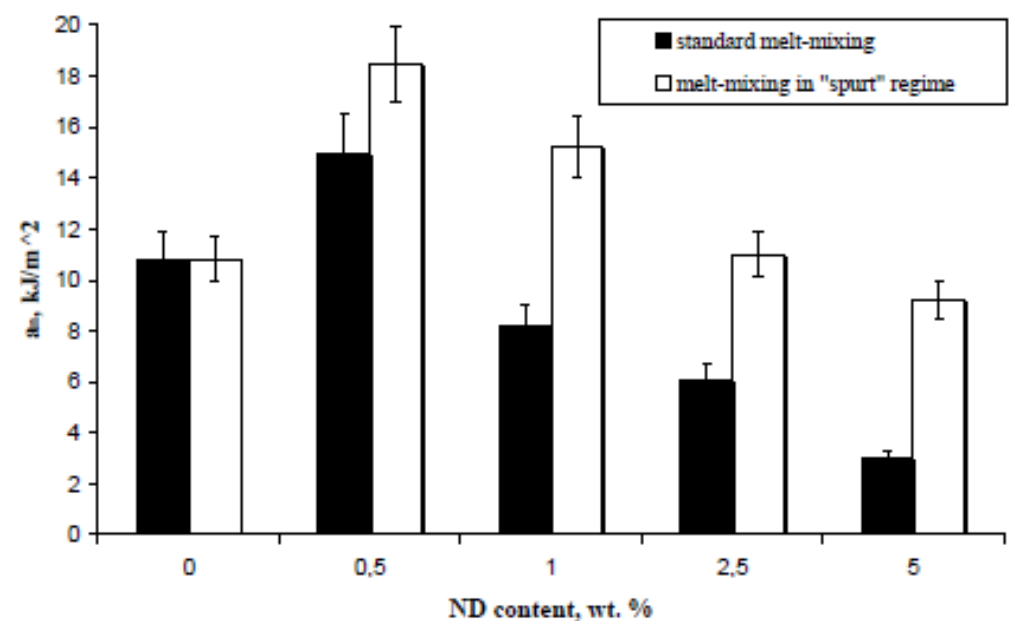

Fig. 39. Izod impact strength of PSF/ND composites manufactured by different ways. 
As it seen, already $0.5 \mathrm{wt}$.\% of ND results in increase of impact strength almost in 2 times. Composites prepared by standard melt-mixing possess essentially the worst properties. This can be attributed to poor uniformity of the ND distribution in the polymer matrix.

\subsection{Tribological properties}

The effect of ND addition to PSF on sliding coefficient of friction is shown in Fig.40. Nanocomposites with ND exhibits a much less steady state coefficient of friction compared with the neat PSF.

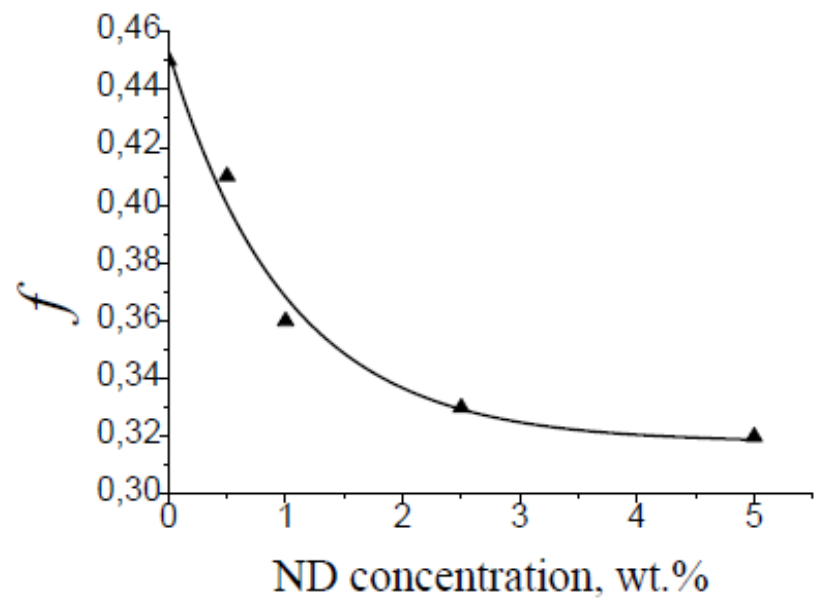

Fig. 40. Friction coefficient of PSF and PSF-ND nanocomposites. Wear conditions: sliding velocity $-1 \mathrm{~m} / \mathrm{s}$, duration $-15 \mathrm{~min}$.

Fig.41 shows the micrographs of the abraded surface of polysulfone and polysulfone nanocomposites (scaled up area of extrudate). By incorporating ND the wear is significantly reduced. Hard nanodiamond particles provide protection and prevent the polymer matrix from severe wear.

a

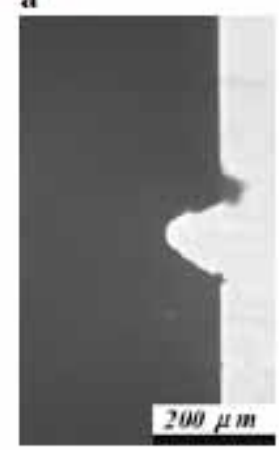

b

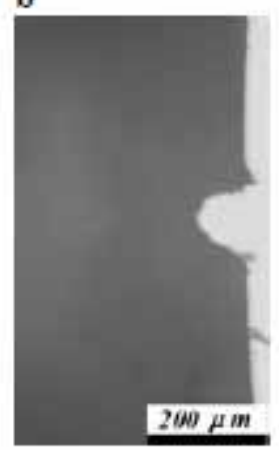

c

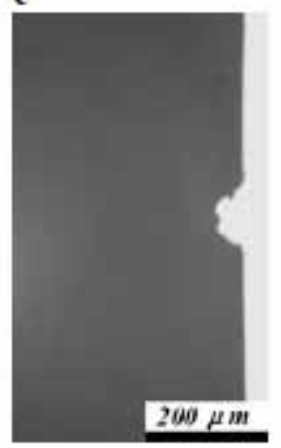

d

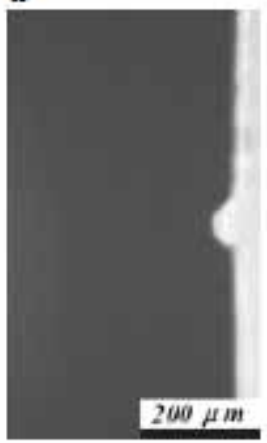

Fig. 41. Micrographs of the worn surfaces of the PSF-ND nanocomposites: neat PSF (a), PSF $/ 1$ wt. \% ND (b), PSF/ 2.5 wt.\% ND (c), PSF/ 5 wt.\% ND (d). Wear conditions: sliding velocity $-1 \mathrm{~m} / \mathrm{s}$, duration $-15 \mathrm{~min}$. 
Brief review of mechanical properties we would like to finish by characteristics of nanocomposites containing modified ND. The corresponding data are shown in Fig.42.

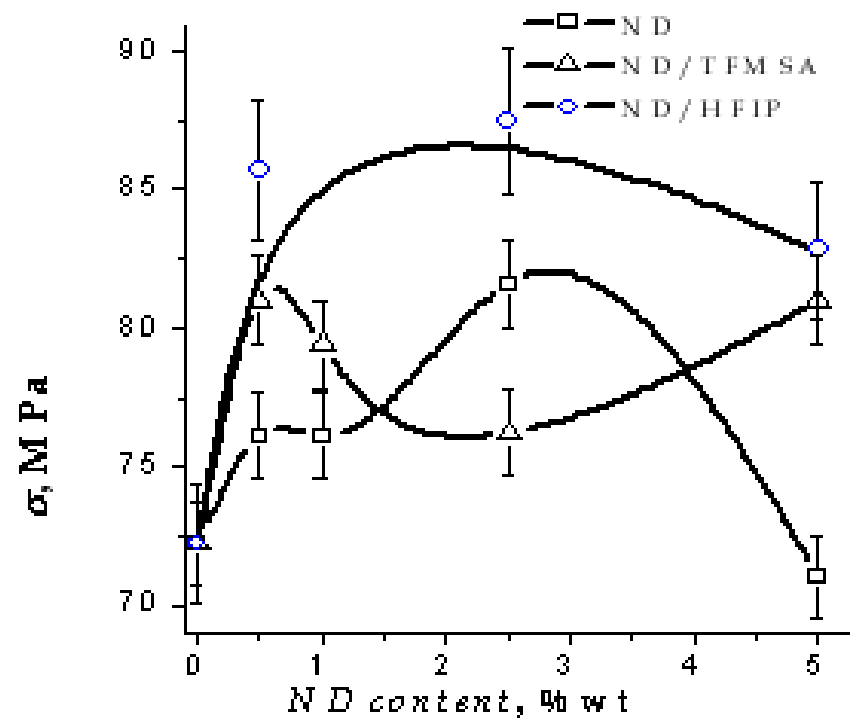

Fig. 42. Concentration dependences of the tensile yield stress for the neat and modified ND.

Essential improvement of mechanical characteristic is observed for ND modified by hexafluoroisopropanol. Namely this reagent leads to blocking carboxyl and carbonyl groups on the surface of ND particles. The similar effect takes place also for elastic modulus. Diminishing of interacting groups on the surface can be reached as correct choice of mechanical mixing regime, as chemical modification. However, due to its simplicity the first way looks more attractive.

\section{Conclusions}

The main focus of this Chapter was targeted on novel approaches to compatibility of particles with polymer matrices and detail analysis of rheology-morphology interrelationships. Rather attractive method of mixing based on knowledge of rheological behavior of polymers at high shear rates was proposed and studied. The major fraction of ND around $40 \mathrm{~nm}$ and absence of agglomerates of micron dimensions allow us to recommend this method for industrial application. For successful processing of nanocomposite precursors interrelationships between rheological properties and stream morphology is extremely important and depending on the task, it is possible to obtain either chaotic or regular distribution of filler particles. These data were obtained by combining rheological and optical or structural methods. Hypothesis over the main driving force of particles ordering based on regular elastic instability of polymer matrix causing ordering of particles was proposed. Mechanical characterization confirms the strong influence of dimensional homogeneity of particles on final properties of nanocomposites. 


\section{References}

Abramowitz M. and Stegun I., "Handbook of Mathematical Functions", chapter 3.2.4. page 11, Dover Publications Inc., ISBN: 0486612724

Detkov, P.Y., Popov, V.A., Kulichikhin V.G., Chukhaeva S.I. (2007). Development of composite materials based on improved nanodiamonds, In Molecular building blocks for nanotechnology, Ali Mansoori, G., George, T.F., Assoufid, L., Zhang, G., pp.29-43, Springer Science+Business Media, LLC, ISSBN: 0-387-39937-2

Dreval, V.E., Khairetdinov, F.N., Litviniv, V.A., Kerber, M.L., Kulichikhin, V.G. (1995). Polymer Sci., Vol.27, №1, pp. 79-86, ISSN:0965-545X

Flory, P.J. (1956). Phase diagrams of rod-like polymer solutions. Proc. Roy. Soc., Ser. A, Vol. 234, No. , pp. 73-89

Jain, S.; Goossens, J.G.P.; Peters, G.W.M.; van Duin, M. \& Lemstra, P.J. (2008). Strong decrease in viscosity of nanoparticle-filled polymer melts through selective adsorption. Soft Matter, Vol. 4, No. , pp. 1848-1854, ISSN: 1539-445X

Larson, R.G. (1992). Instabilities in viscoelastic flows. Rheol. Acta, Vol. 31, No. 3, pp. 213-263, ISSN:0035-4511

Karbushev, V.V.; Konstantinov, I.I.; Semakov A.V.; Parsamyan I.L.; Kulichikhin V.G. \& Popov V.A. (2007). Polymer composite reinforced with nanodiamonds and clay, In: Mater. Res. Soc. Symp. Proc. Volume 1007, Barbé, C.; Laine, R.M.; Sanchez, C. \& Schubert, U.V. (Ed.), pp. 1-317, Warrendale, PA, ISBN: 978-1-55899-967-1

Karbushev, V.V.; Konstantinov, I.I.; Kulichikhin, V.G.; Khodzhaeva, V.L. \& Popov, V.A. (2008). Polymer-nanodiamond composites: preparation, and characterization, Proceedings of the $3^{\text {rd }}$ International Symposium "Detonation Nanodiamonds: Technology, Properties and Applications", pp. 73-78, ISBN: 978-5-93634-027-9, St.-Petersburg, Russia, July 2008, Ioffe Physico-Technical Institute, St.-Petersburg

Konstantinov, I.I.; Karbushev, V.V.; Semakov, A.V. \& Kulichikhin, V.G. (2009). Combining Carbon and Polymeric Particles in an Inert Fluid as a Promising Approach to Synthesis of Nanocomposites. Russian Journal of Applied Chemistry, Vol. 82, No. 3, pp. 483-487, ISSN: $1070-4272$

Karbushev, V.V.; Konstantinov, I.I.; Parsamyan, I.L.; Kulichikhin, V.G.; Popov, V.A. \& George T.F. (2009). Preparation of polymer-nanodiamond composites with improved properties. Advanced Materials Research, Vol. 59, pp. 275-278, ISSN: 10226680 (print version)

Kulichikhin, V.G.; Plotnikova, E.P.; Tereshin, A.K.; Subbotin, A.V. \& Tsamalashvili, L.A. (2000). Rheological and Interfacial Properties of Homophase and Heterophase Anisotropic Polymers. Polymer Science, Ser. C, Vol. 42, No. 2, pp. 129-154, ISSN:0965-545X

Kulichikhin, V.G.; Plotnikova, E.P.; Subbotin, A.; Plate, N.A. (2001). Specific rheology morphology relationships for some blends containing LCPs. Rheol. Acta, Vol. 40, No. , pp. 49-59, ISSN: 0035-4511

Kulichikhin, V.G.; Makarova, V.V.; Tolstykh, M.Yu. \& Vasil'ev, G.B. (2010). Phase Equilibria in Solutions of Cellulose Derivatives and the Rheological Properties of Solutions in Various Phase States. Polymer Science, Ser. A, Vol. 52, №11 (in press), ISSN:0965545X 
Kwolek, S.-L. (1972). Optically Anisotropic Aromatic Polyamide Dopes, US Patent 3671542

R.G. Larson (1991). Review: Instabilities in viscoelastic flows. Rheol. Acta, Vol. 31. No 3. pp. 213-263, ISSN: 0035-4511

Malkin, A.Ya. (2006). Flow instability in polymer solutions and melts. Polymer Science, Ser. C, Vol. 48, No. 1, pp. 21-37, ISSN: 1811-2382

Mackay, M.E.; Dao, T.T.; Tutega, A.; Ho, D.L.; van Horn, B.; Kim, H.-C. \& Hawker, C.J. (2003). Nanoscale effects leading to non-Einstein-like decrease in viscosity. Nature Materials, Vol. 2, No. , pp. 762-766, ISSN: 1476-4660

Malkin, A.; Askadsky, A.; Chalykh, A. \& Kovriga, V. (1983). Experimental methods of polymer physics (Measurements of mechanical properties, viscosity, and diffusion). (Ed. A.Ya. Malkin) Prentice-Hall Inc. Englewood Cliffs, N.J., USA. Library of Congress Catalog Card Number 83-60908

Makarova, V.V. (2007). Masstransfer in vicinity of interfaces in polymer-polymer systems. PhD Thesis. Moscow, A.V.Topchiev Institute of Petrochemical Synthesis

Makarova, V.V., Kulichikhin, V.G., Picken, S-J. Mendes, E. (2010). Rheo-X-ray study of complex liquids in shear flow, Proceedings of Pacific Rim Conference on Rheology5, Sapporo, August 1-5

Papkov, S.P. \& Kulichikhin, V.G. (1977). Liquid-crystal state of polymers. Khimiya, Moscow (in Russian)

Papkov, S.; Kulichikhin, V. \& Kalmykova, V. (1974). Rheological properties of anisotropic poly(p-benzamide) solutions. J. Polym. Sci., Polym. Phys. Ed., Vol. 12, No. , pp. 17531764, ISSN: 0887-6266

Pinnovaia, T.J. \& Beall, G.W. (Ed.) (2000). Polymer-Clay Nanocomposites, John Wiley \& Sons Ltd, ISBN: 0-471-63700-9, Chichester, England

Ruijter, C.; Mendes, E.; Boerstoel, H. \& Picken, S.J. (2006). Orientational order and mechanical properties of poly(amide-block-aramid) alternating block copolymer films and fibers. Polymer, Vol. 47, pp. 8517-8526, ISSN: 0032-3861

Semakov, A.V. \& Kulichikhin, V.G. (2009). Self-assembly and elastic instability in polymer flow. Polymer Science, Ser. A, Vol., No. 11-12, pp. 1313-1328, ISSN: 0965-545X

Shenderova, O.A.; Zhirnov, V.V. \& Brenner, D.W. (2004). Carbon nanostructures. Critical Reviews in Solid State and Materials Sciences, Vol. 27, No. 3-4, pp. 227-356, ISSN: 10408436

Schmidt G., Nakatani, A.I., Butler, P.D., Karimk, A., Han, C.C. (2000). Macromolecules, Vol.33, №20, pp.7219-7222. ISSN: 0024-9297

Viale, S.; Best, A.S.; Mendes, E. \& Picken, S.J. (2005). Formation of molecular nematic liquid crystal phase in poly(p-sulfophenylene sulfotherephtalamide), Chemical Communications, Vol. , № 12, pp. 1528-1530, ISSN: 1359-7345

Vinogradov, G.V. \& Malkin, A.Ya. (1966). Comparative description of the peculiarities of deformation of polymer and plastic disperse systems. Rheol. Acta, Vol. 5, No. 3, pp. 188-193, ISSN: 0035-4511

Vinogradov, G.V., Ivanova, L.I. (1968).Wall slippage and elastic turbulence of polymers in rubbery state, Rheol. Acta, Vol.7, №3 pp.243-254, ISSN: 0035-4511 
Yang, X., Wang, S-Q., Halasa A., Ishida H. (1998). Fast flow behavior of highly entangled monodisperse polymers. 1. Interfacial stick-slip transition of polybutadiene melts, Rheol.Acta, Vol.37, №5, pp.415-423, ISSN: 0035-4511

Yarin, A.L., Graham, M.D., (1998). A model for slip at polymer/solid interfaces, J.Rheol., Vol.42, №6, p.1491-1505; ISSN: 0148-6055 